\title{
DECONSTRUCTING MINDFULNESS AND CONSTRUCTING MENTAL HEALTH: UNDERSTANDING MINDFULNESS AND ITS MECHANISMS OF ACTION
}

\author{
Kimberly A. Coffey
}

A dissertation submitted to the faculty of the University of North Carolina at Chapel Hill in partial fulfillment of the requirements for the degree of Doctorate of Philosophy in the Department of Psychology (Clinical Psychology).

Chapel Hill

2008

Approved by:

Barbara Fredrickson, Ph.D.

Karen Gil, Ph.D.

Marilyn Hartman, Ph.D.

David Thissen, Ph.D.

Erica Wise, Ph.D. 
(C) 2008

Kimberly A. Coffey

ALL RIGHTS RESERVED 


\begin{abstract}
KIMBERLY A. COFFEY: Deconstructing Mindfulness and Constructing Mental Health: Understanding Mindfulness and Its Mechanisms of Action (Under the direction of Barbara Fredrickson, Ph.D. \& Marilyn Hartman, Ph.D.)
\end{abstract}

Mindfulness is associated with improved mental health, but the mechanisms by which it exerts its beneficial effects are currently unclear. The sequence of two studies presented here attempted to better understand the composition of mindfulness, as a construct, and explored potential mechanisms by which it might influence mental health. These studies examined mindfulness as a naturally-varying, individual difference in a non-clinical sample.

The first study examined both the factor structure of current mindfulness and emotion regulation measures and the relationships among these factors. Factor analysis results indicated that the terms "mindfulness" and "emotion regulation" refer to heterogeneous and overlapping constructs, and may be more accurately thought of as present-centered attention, acceptance of experience, clarity about one's internal experience, and the ability to manage negative emotions. Furthermore, results from a structural equation model that combined the factor structure identified above with a path analysis suggested that clarity and the ability to manage negative emotions may be sequelae of the two most commonly-recognized components of mindfulness, present-centered attention and acceptance of experience. 
The second study expanded upon results from the first study to explore four potential mechanisms of action in the relationship between mindfulness, redefined after the first study as present-centered attention and acceptance of experience, and mental health, which included both psychological distress and flourishing mental health. The four potential mechanisms of action examined were clarity about one's internal life, the ability to manage negative emotions, non-attachment (or the extent to which one's happiness is independent of specific outcomes and events), and rumination. Results confirmed the importance of these four constructs as mediators in the relationship between mindfulness and mental health. One aspect of mindfulness, present-centered attention, exhibited a complex and paradoxical relationship with psychological distress, whereby an increased tendency to observe one's present-moment experience was directly associated with greater psychological distress, but indirectly associated with diminished distress via attention's salubrious impact on intervening variables in the model. 


\section{TABLE OF CONTENTS}

Page

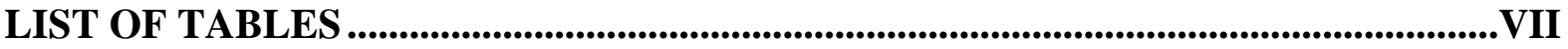

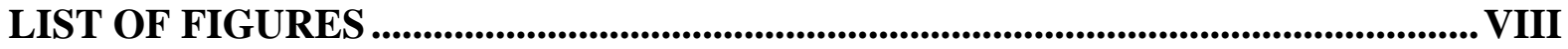

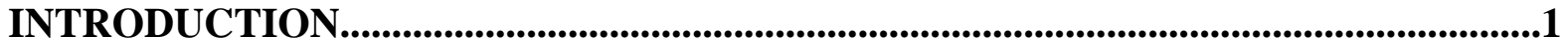

MiNDFULNESS MEASURES ...................................................................................

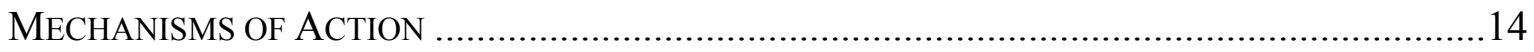

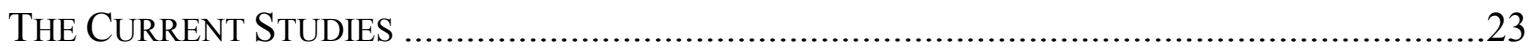

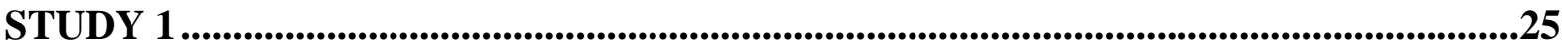

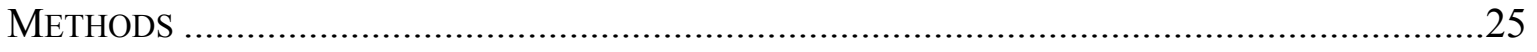

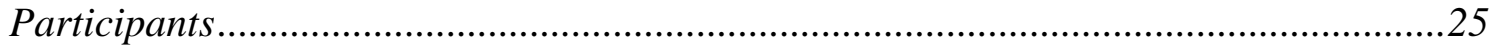

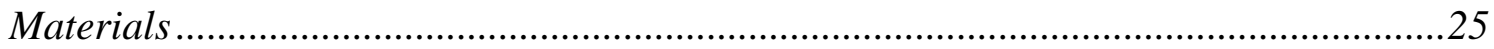

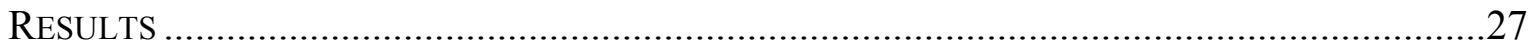

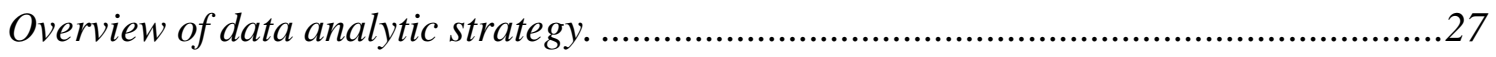

Step 1: Confirmatory Factor Analysis for Original Mindfulness and Emotion Regulation

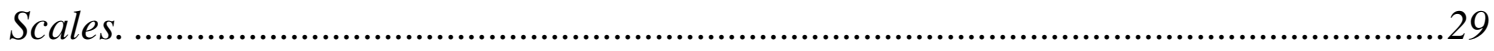

Step 2: Exploratory Factor Analyses..................................................................30

Step 3: Confirmatory Factor Analyses for the Four Factors Identified in the Exploratory

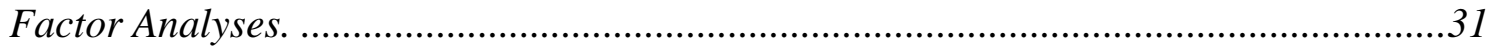

Step 4: Path Analysis Examining the Relationships among the Common Factors..........33

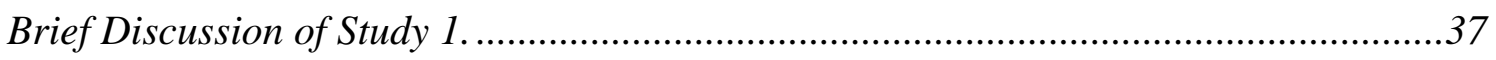

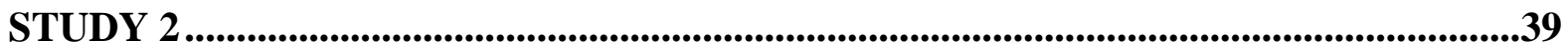

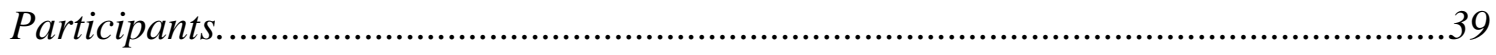


Materials.

RESULTS

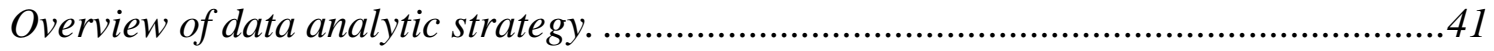

Step 1: Confirmation of Factor Structure from Study 1. ..........................................43

Step 2: Mechanisms of Action in the Relationship between Mindfulness and Psychological Distress.

Step 3: Mechanisms of Action in the Relationship between Mindfulness and Flourishing Mental Health

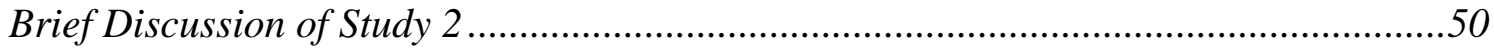

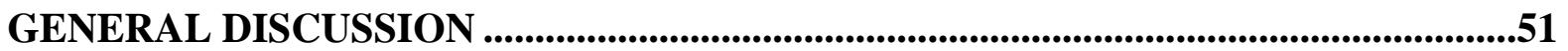

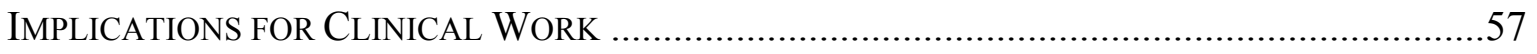

LIMITATIONS AND FUTURE DIRECTIONS .............................................................59

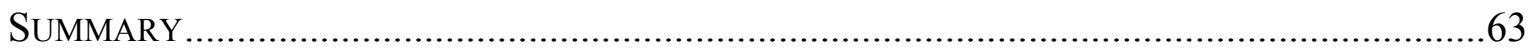

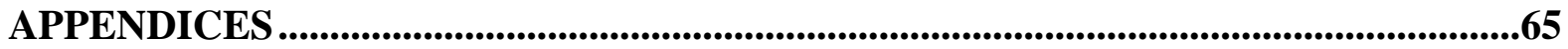

ApPendix A: Additional Models Tested In StUdy 1 .........................................65

ApPEndix B: Additional AnAlyses with AtTENTION AND ACCEPTANCE ......................68

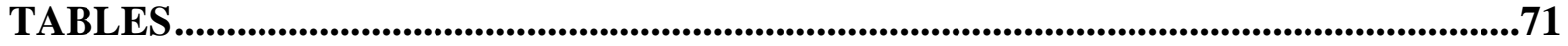

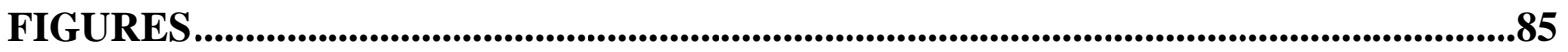

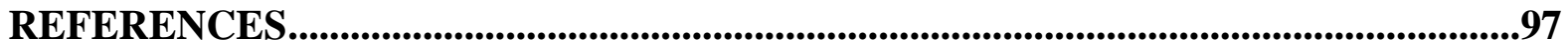




\section{LIST OF TABLES}

Page

Table 1. Descriptive Statistics and Indicator Inter-correlations (Study 1) ........................... 72

Table 2. Exploratory Factor Analysis with Three Factors

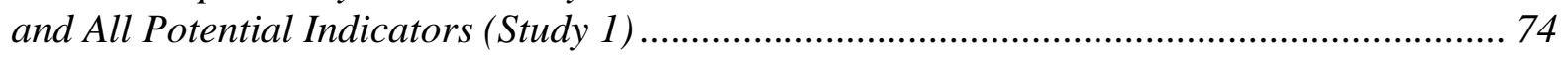

Table 3. Exploratory Factor Analysis with Four Factors

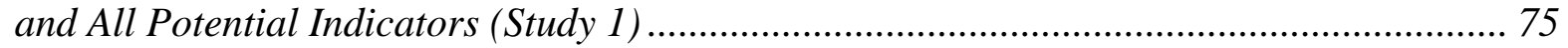

Table 4. Exploratory Factor Analysis Excluding the FFMQ

Acting with Awareness Subscale (Study 1) .................................................................... 76

Table 5. Factor Inter-correlations for Acceptance, Clarity, and

Negative Emotion Regulation (Study 1) ....................................................................... 77

Table 6. Descriptive Statistics and Indicator Inter-correlations (Study 2) ......................... 78

Table 7. Confirmatory Factor Analysis Standardized Factor Loadings (Study 2)............... 80

Table 8. Factor Inter-correlations for Acceptance, Clarity, and

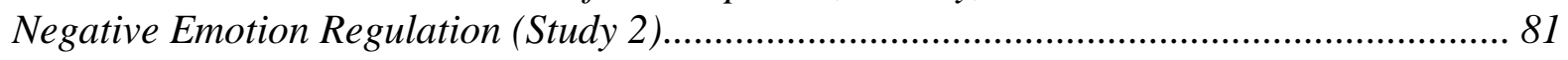

Table 9. Indirect Effects (Standardized) of Each Factor on Other Factors (Study 2)........... 82

Table 10. Total Effects (Standardized) of Each Factor on Other Factors (Study 2)............. 83

Table 11. Total Effects (Standardized) of Each Factor on Other Factors

when Both Psychological Distress and Positive Psychological Adjustment

are Included in the Model (Study 2) 


\section{LIST OF FIGURES}

Figure 1. Confirmatory Factor Analysis for Four Factors Identified in Exploratory Factor Analysis (Study 1)

Figure 2. Confirmatory Factor Analysis for Acceptance, Clarity, and

Negative Emotion Regulation (Study 1).

Figure 3. Relationships among the Factors (Study 1) .................................................... 87

Figure 4. Full Model with All Possible Pathways (Study 2)............................................ 88

Figure 5. Full Model with All Hypothesized or Significant pathways (Study 2).................. 89

Figure 6. Full Model with Flourishing Mental Health as the Final Variable and all Possible Pathways (Study 2)...

Figure 7. Full Model with Flourishing Mental Health as the Final Variable and all Significant Pathways (Study 2)

Figure 8. Full Model with Both Psychological Distress and Flourishing Mental Health as Outcome Variables (Study 2).

Figure 9. Exploratory Model 1: Incorporating the FFMQ Acting with Awareness Subscale into the Model (Study 1)

Figure 10. Exploratory Model 2: Incorporating the FFMQ Acting with

Awareness Subscale into the Model (Study 1).

Figure 11. Exploratory Model 3: Incorporating the FFMQ Acting with

Awareness Subscale into the Model (Study 1). 95

Figure 12. Exploratory Model 4: Incorporating the FFMQ Acting with Awareness Subscale into the Model (Study 1). 


\section{INTRODUCTION}

Recent years have witnessed a surge of popular and academic interest in the psychological benefits of mindfulness. Mindfulness is believed to facilitate insight into one's mental and emotional life (Kyabgon, 2001), improve the ability to recognize and overcome automatic reactions and habitual thought patterns (Deikman, 1966), and enhance wisdom, equanimity, and life satisfaction (Kabat-Zinn, 1994). Time magazine (Begley, 2007; Cullen, 2006), Newsweek (Kalb, 2004; Ornish, 2006), National Geographic (Garfinkel, 2006), and the New York Times (Jenkins, 2007) have all published stories on meditation and mindfulness-based practices in the past three years. Mindfulness is being used to enhance functioning in populations as varied as baseball pitchers (Jenkins, 2007), Wall Street investors (Cullen, 2006), employees at a biotechnology firm (Davidson et al., 2003), medical and premedical students (Shapiro, Schwartz, and Bonner, 1998), college students suffering from math anxiety (Zettle, 2003), and nondistressed couples looking to improve their relationship (Carson, Carson, Gil, \& Baucom, 2004). A growing number of treatments for psychological distress incorporate mindfulness, as well, including mindfulness-based stress reduction (MBSR; Kabat-Zinn, 1990), mindfulness-based cognitive therapy (MBCT; Segal, Williams, \& Teasdale, 2002), acceptance and commitment therapy (ACT; Hayes, Strosahl, \& Wilson, 1999) and dialectical behavior therapy (DBT; Linehan, 1993). These interventions have been found to reduce many forms of distress, including anxiety (Kabat-Zinn et al., 1992), depression (Kabat-Zinn et al., 1992; Shapiro et al., 1998; Speca, Carlson, Goodey, \& Angen., 2000), depressive relapse (Teasdale et al., 2000), anger (Speca et al., 2000), 
Attention Deficit Hyperactivity Disorder (Zylowska et al., 2006), and parasuicidal behavior (Linehan, Armstrong, Suarez, Allmon, \& Heard, 1991).

Despite this burgeoning interest in mindfulness and its possible applications, it is a very new research field, and has yet to achieve a consensual definition (Dimidjian \& Linehan, 2003). To address this issue, an interdisciplinary team of mindfulness and meditation researchers convened to develop an operational definition for the construct. In the publication that resulted from this meeting, Bishop et al. (2004) argue that mindfulness encompasses both an attentional and an acceptance-based component. The attentional component pertains to the ability to intentionally regulate attention, which is honed by deliberate and sustained focus on thoughts, feelings, physical sensations, and other stimuli as they occur in the present moment. The acceptance-based ${ }^{1}$ component involves maintaining an attitude of openness and receptivity to these experiences, rather than ignoring or minimizing them, particularly when they are unpleasant. Present in this conceptualization is a sense that mindfulness consists of both an action (i.e., attending to present-moment experience) and a specific attitude with which the action is performed (i.e., nonjudgmental, accepting orientation towards experience). These two components are common to almost all definitions for mindfulness.

Different conceptualizations of mindfulness include other elements, in addition to present-centered attention and nonjudgmental orientation towards experience. For example, in DBT, mindfulness encompasses the two elements described above (i.e., observation of experience and nonjudgmental orientation towards experience), as well as four additional 
elements. These include describing experience with words, fully participating in experience, completing activities "one-mindfully," and focusing on effective behavior (Linehan, 1993). Describing experience with words involves verbal articulation of one's experience. For example, DBT clients might be encouraged to label the particular emotion they are experiencing, such as sadness or anger. Participating in experience can be contrasted with doing things in a half-hearted or resistant manner. It involves genuinely trying to contribute to or take part in an experience. One-mindful behavior involves doing only one thing at a time. For example, a DBT client might select one behavior, such as cooking dinner, and do only that, rather than simultaneously cooking, watching television, and talking on the phone. Effective behavior accomplishes a previously-specified goal. It can be contrasted with engaging in behavior that driven by a sense of what "should" work. For example, a client might typically use an angry or belligerent voice to request that his or her partner complete a household task because s/he feels the partner completes an insufficient portion of the housework. When the client does this, the partner becomes irritated, a fight ensues, and the client him- or herself often completes the task. Engaging in "effective" behavior involves identifying a goal (e.g., partner completes task), and then engaging in behavior that is most likely to accomplish this goal (e.g., ask nicely).

It should be noted, first, that DBT was designed as a skills-based intervention. The mindfulness skills described above are intended to help clients learn mindfulness. It is therefore possible that some DBT mindfulness skills may be better understood as techniques that assist clients in learning mindfulness, rather than representing mindfulness, per se. For

\footnotetext{
${ }^{1}$ A common misconception about the term "acceptance" in this context is that it connotes passivity or resignation; this is a misunderstanding (Breslin, Zack, \& McMain, 2002). Acceptance refers to acknowledgement that one is having a given experience, even if the experience is unpleasant, and can be
} 
example, it would be difficult to describe one's experience in language without first attending to it. Thus, it is useful to teach present-centered attention or observation of experience to clients by encouraging them to label their experience with language. Nonetheless, the ability to use language to describe experience may be a skill that is facilitated by observing one's experience, rather than an expression of the same construct.

Second, it should also be noted that DBT was designed to help participants diminish their psychological distress. Some aspects of mindfulness in DBT may therefore be useful for managing psychological distress, but may not reflect historic conceptualizations of mindfulness (e.g., effective behavior). In DBT, mindfulness is employed to help clients collect information about themselves and their environments, which they can use to more effectively manage their emotions and distress. In this context, the boundary between activities that assist with mindfulness and those that assist with other abilities related to managing distress, such as emotion regulation, can become blurred. This issue is discussed in greater detail below.

Some conceptualizations of mindfulness also include the practitioner's intention, or objective, for his or her mindfulness practice (Kabat-Zinn, 1994; Shapiro, Carlson, Astin, \& Freedman, 2006). Classic intentions in Eastern contemplative practice include enlightenment, liberation from suffering, and the liberation of all sentient beings. To this author's knowledge, advocates of this position have not delineated whether some intentions are incompatible with mindfulness (e.g., improving attentional control and nonjudgmental thinking so one can more calmly and effectively engage in nefarious acts).

contrasted with denial or avoidance. 
The intentional element limits mindfulness to something that occurs exclusively in the context of a particular practice. This circumscribed definition for mindfulness conflicts with the belief that mindfulness exists as a naturally-occurring individual difference, which can be enhanced with a formal mindfulness practice (Baer, 2003; Brown \& Ryan, 2003; Brown \& Ryan, 2004). Much of the research literature on mindfulness (e.g., Brown \& Ryan, 2003; Baer, Smith, \& Allen, 2004), considers that mindfulness is a particular kind of attention that exists apart from the deliberate attempt to enhance it. The possibility that mindfulness might function differently in those with formal mindfulness training than in those without formal training has not been fully explored.

\section{$\underline{\text { Mindfulness Measures }}$}

As interest in understanding and using mindfulness in psychological interventions has grown, interest in inexpensive, self-report measures for the construct has grown as well. This endeavor is plagued by two prominent difficulties. First, as each of the preceding conceptualizations of the construct makes clear, different researchers and theorists understand the construct differently. As a result, mindfulness measures could conceivably encompass as few as two (Bishop et al., 2004) or as many as six (Linehan, 1993) different facets. These measures could also assess behaviors as molecular as where a person's attention is at one point in time (e.g., attention or observation of experience), or as molar as a complex sequence of actions and its consequences (e.g., effective behavior).

A second difficulty is that if mindfulness is a multi-faceted construct, as most conceptualizations contend, the relationships among these facets are unclear. As mentioned above in the context of the different DBT mindfulness skills, it would be difficult for 
someone to describe his or her experience without first attending to it. Similarly, it would be difficult for someone to act effectively in a difficult situation without observing and nonjudgmentally recognizing the situation as it is. This admits the possibility that some constructs might reflect a purer, and more consensually-recognized, form of mindfulness and others might be sequelae of these constructs. The relations among different, proposed aspects of mindfulness have not been theoretically developed.

The existing self-report mindfulness questionnaires reflect these difficulties in the research literature. There are multiple measures that each assess slightly different constructs. Even when multiple constructs are represented within a measure, the potential relationships among these constructs have not been articulated.

One of the most widely-used mindfulness measures is the Mindful Attention Awareness Scale (MAAS; Brown and Ryan, 2003). The MAAS is intended to measure one of the primary components in most definitions for mindfulness, present-centered attention. MAAS questions examine attention to and awareness of present-moment experience by assessing the phenomenological and behavioral correlates of its opposite, absent-mindedness. The measure includes items associated with doing things without paying attention, such as breaking or spilling things because of carelessness, or forgetting a person's name as soon as one has been introduced. Brown and Ryan (2003) argue that mindless states are more familiar to most people, and a measure for mindlessness is therefore more accessible and has 
more predictive power for most people than would a measure for mindfulness. ${ }^{2}$ Indirect measurement of present-centered attention, via absent-mindedness and automated behavior, may make the MAAS a more accurate measure for "one-mindful" behavior than for presentcentered attention, however. In constructing the MAAS, Brown and Ryan (2003) measured automated, unaware behavior and eliminated questionnaire items that failed to load on this factor.

In contrast to the other mindfulness measures discussed below, the MAAS does not include items related to acceptance of one's experience. An original version of the MAAS included two factors: one for absent-mindedness and a second factor for an attitude of acceptance. Although factor analyses supported the existence of these two distinct factors, the absent-mindedness factor was as highly correlated with a variety of psychological adjustment measures as were the absent-mindedness and acceptance factors combined. Furthermore, convergent, discriminant, and criterion validity research indicated that the acceptance factor provided no additional explanatory power in predicting performance on these measures of psychological adjustment. The researchers concluded that the absentmindedness factor subsumes the acceptance factor. The acceptance factor and its associated questions were subsequently dropped from the MAAS (Brown \& Ryan, 2004).

The MAAS has been found to predict multiple measures for mental health. MAAS scores were positively correlated with measures of eudaimonic well-being, such as feelings of autonomy, competence, and positive relations with others, and negatively correlated with

\footnotetext{
${ }^{2}$ An alternative version of the MAAS re-phrased each question to measure the presence of aware and deliberate behavior (Brown \& Ryan, 2003). This version was related to a variety of psychological measures in the hypothesized directions; the magnitude of the relationship was often weaker, however. The greatest differences between the MAAS and its alternative, positively-worded measure were evident for measures of psychological distress, such as anxiety, rumination, depression, and negative affect. For these measures, the negatively-
} 
anxiety, hostility, depression, self-consciousness, and impulsivity (Brown \& Ryan, 2003). In addition, individuals who meditate scored higher on the MAAS than matched community controls, and MAAS scores were related to the number of years respondents had practiced meditation (Brown \& Ryan, 2003).

Unlike the MAAS, the Kentucky Inventory of Mindfulness Skills (KIMS; Baer, Smith, \& Allen, 2004) was developed to measure multiple hypothesized components of mindfulness and intentionally contains multiple factors. In developing the measure, the authors relied heavily on the conceptualization of mindfulness presented in DBT, although they note that the aspects of mindfulness assessed by their measure are consistent with other accounts of mindfulness, as well. The KIMS assesses the frequency with which the respondent observes his or her experience, accepts his or her experience, describes experience, and acts with awareness versus on automatic pilot. The first two factors correspond to the attentional and nonjudgmental aspects of mindfulness, respectively, that are common to almost all mindfulness definitions. The third measures the ability to label experience with language, and reflects the DBT mindfulness skill associated with this ability. The last jointly assesses the DBT skills associated with "participating" in experience and acting "one-mindfully." Exploratory and confirmatory factor analyses confirmed this factor structure.

Baer, Smith, and Allen (2004) administered the KIMS to samples of undergraduate students ( $n=205$ and 215, respectively) and discovered that these four factors exhibited different patterns of relationship with each other and with psychological distress, which suggests that they assess distinct psychological phenomena. In addition, the Observation of Experience subscale exhibited a particularly surprising pattern of relationships. The

worded MAAS was often correlated more highly than was the positively-worded alternative measure. 
Acceptance, Describe, and Acting with Awareness subscales were all significantly and positively correlated with each other, with correlations ranging between 0.22 and 0.34 . The Observation of Experience subscale, however, was positively correlated with the Describe subscale, not significantly correlated with the Acting with Awareness subscale, and significantly negatively correlated with the Acceptance subscale. Furthermore, all subscales except Observation of Experience were significantly negatively correlated with psychological distress. In contrast, Observation of Experience was not significantly related to psychological distress. Thus, although observation of experience or present-centered attention is central to all mindfulness definitions, this aspect of mindfulness did not exhibit the positive relationship to acceptance of experience and negative relationship to psychological distress that theory (e.g., Kabat-Zinn, 1990; Kabat-Zinn, 1994) would predict.

A third mindfulness measure, the Freiburg Mindfulness Inventory (FMI; Buchheld, Grossman, \& Wallach, 2001), differs from the MAAS and KIMS in that it was originally developed for use with experienced meditators. Questions on the FMI pertain to observation of internal and external stimuli, acceptance of and nonjudgmental reactions to experiences, and more cognitively-based questions that assess beliefs that are part of Buddhist philosophy, such as recognition of the ephemeral nature of life or the way in which people often create their own suffering. The authors argue that the FMI assesses one over-arching construct, although there are some difficulties with the statistical analyses they provide to support this claim (Walach, Buchheld, Buttenmuller, Kleinknecht, \& Schmidt, 2006). The authors attempted to explore the possible existence of common factors among their questionnaire items by using a principal components analysis, which is more appropriate for explaining the maximum amount of variance in measured variables than it is for explaining covariance 
among measured variables. An exploratory factor analysis would have been more appropriate, given the question of interest. The authors also performed a varimax rotation, which imposes orthogonal factors. Any common factors are not orthogonal, as subsequent analyses revealed. The authors' principal components analysis with varimax rotation resulted in a four factor solution. As a second step, the authors performed an oblique rotation on their four factor solution, which revealed correlations ranging from 0.48 to 0.60 between the factors. Walach et al. (2006) interpret these correlations as implying one, general factor. Factor inter-correlations of this size could easily be used to argue in favor of four separate, albeit related, constructs, however. Furthermore, it is unclear whether the proposed factor structure would fit well if it were tested in a confirmatory factor analysis, or whether an exploratory factor analysis with oblique rotation would reveal the same solution. For these reasons, the authors' conclusion that the FMI assesses one, general construct is considered to be suspect.

Although the FMI was originally designed for use with experienced meditators, Baer, Smith, Hopkins, Krietemeyer, and Toney (2006) administered the FMI to a sample of undergraduate students $(n=613)$, and confirmed that scores on the FMI were positively correlated with meditation experience, emotional intelligence, and self-compassion and negatively correlated with psychological symptoms, difficulties in emotion regulation, and alexithymia. The alpha coefficient also suggested good internal reliability. These data suggest that although the FMI was designed for use with experienced meditators, it may be appropriate to use the measure in samples with no or little formal mindfulness training.

The MAAS, KIMS, and FMI each originate in slightly different conceptualizations of mindfulness, and emphasize different hypothesized aspects of the construct. In particular, the 
FMI explicitly incorporates beliefs associated with the Eastern philosophical traditions in which mindfulness practices originated. The KIMS and the MAAS move away from Eastern contemplative beliefs and assess more concrete, cognitive and behaviorally-driven versions of mindfulness. The KIMS assesses five of the six skills taught under the rubric of mindfulness training in DBT. (Only the "effective behavior" skill is not included in the KIMS. The "one-mindful" and "participate fully" skills are jointly assessed as Acting with Awareness.) The MAAS measures day-to-day experiences associated with doing things in a mindless or automated way.

To explore the relationships among these different conceptualizations of the construct, Baer et al. (2006) combined several existing self-report trait-level mindfulness questionnaires, including the MAAS, the KIMS, the FMI, and two unpublished measures, the Cognitive and Affective Mindfulness Scale (Feldman, Hayes, Kumar, \& Greeson, 2004) and the Mindfulness Questionnaire (Chadwick, Hember, Mead, Lilley, \& Dagnan, 2005). The researchers examined these measures in an undergraduate sample $(n=613)$, which largely had no formal mindfulness training. An exploratory factor analysis with oblique rotation revealed the presence of five factors, which the researchers described as: Observation of experience; a nonjudging or accepting orientation towards experience; acting with awareness versus functioning on automatic pilot; the ability to describe experience; and non-reactivity to inner experience. The last factor examined the respondent's ability to tolerate internal distress without feeling overwhelmed by it, becoming stuck in it, or acting out in response to it. Questions from the MAAS loaded exclusively on the Acting with Awareness factor. Baer et al. (2006) tested and confirmed this factor structure using a confirmatory factor analysis in an independent sample $(n=268)$. 
Baer et al. (2006) found that the five mindfulness factors were significantly correlated with each other in the expected directions, with the exception of the correlation between the tendency to observe one's experience and refrain from judging experience, which was not significant. This was an unexpected finding, given that these two components are common to almost all definitions for mindfulness (e.g., Bishop et al., 2004; Kabat-Zinn, 1994; Linehan, 1993; Shapiro et al., 2006), but was generally consistent with findings by Baer et al. (2004), who found that these two constructs were negatively correlated in a sample of undergraduates with no formal mindfulness training.

Furthermore, all of the factors except Observation of Experience were significantly and negatively correlated with difficulties with emotion regulation and psychological distress. In contrast, Observation of Experience was unrelated to emotion regulation and positively correlated with psychological distress. This was also an unexpected finding, given the rich history of Buddhist thought on the psychological benefits of mindfulness (Goldstein, 1976; Kyabgon, 2001) and the beneficial impact of mindfulness-based interventions on mental health (e.g., Kabat-Zinn et al., 1992; Shapiro et al., 1998; Speca et al., 2000). Nonetheless, there is some precedent for this finding in Baer, Smith, and Allen's (2004) finding that Observation of Experience and psychological distress were not significantly correlated.

To better understand the role of Observation of Experience in samples with and without mindfulness training, Baer et al. (2006) created a subsample of participants with meditation experience $(n=190)$, and explored the correlations among their five factors in this subsample. They found that the correlation between Observation of Experience and Nonjudging became significant and positive, whereas the other correlations among the other 
factors did not change. In this subsample, the relationship between Observation of Experience and psychological distress became non-significant. This finding suggests that deliberately attending to one's experience may be differentially related to other constructs in those with and without formal mindfulness training.

Baer et al. (2006) used these findings on the factor structure for existing mindfulness questionnaires to create a new mindfulness measure, the Five Factor Mindfulness Questionnaire (FFMQ). The FFMQ is composed of questions from each of the mindfulness questionnaires used in their factor analysis, and includes five subscales for the five factors described above. Questions that did not load cleanly on one of the five factors were not used. The FFMQ reflects the most comprehensive attempt to measure mindfulness currently available.

These studies reveal three important findings relevant to understanding mindfulness as a construct. First, these studies explored mindfulness as a naturally-varying, individual difference in samples with little or no formal mindfulness training (Baer et al., 2004; Baer et al., 2006; Brown \& Ryan, 2003). Results indicated that the measures were generally associated with psychological distress in the expected directions in this population. This suggests that the conceptualizations of mindfulness assessed by these measures can be reliability measured as a naturally-varying individual difference, in the absence of a mindfulness practice, and that some aspects of naturally-varying mindfulness are associated with psychological distress in the expected direction.

Second, aspects of mindfulness appear broadly related to psychological distress, but Observation of Experience, which reflects the attentional component that is common to all mindfulness definitions, was not significantly related to psychological distress in one study 
(Baer et al., 2004) and was positively associated with psychological distress in another (Baer et al., 2006). This surprising finding conflicts with theory about the salubrious impact that present-centered attention can have on mental health (e.g., Kabat-Zinn, 1990; Kabat-Zinn, 1994). It furthermore suggests that whereas some aspects of mindfulness may function in the hypothesized way in the absence of mindfulness training, present-centered attention may not.

Third, consistent with the varied theoretical perspectives from which mindfulness measures are derived, existing measures encompass five distinct constructs (Baer et al., 2006). The include present-centered attention or observation of experience, a nonjudgmental or accepting relationship towards one's experience, the ability to use language to articulate one's experience, a nonreactive relationship towards distressing emotions, and engaging in one-mindful and aware behavior. The relationships among these constructs are not clear.

\section{$\underline{\text { Mechanisms of Action }}$}

Baer et al. (2006) examined the five facets of mindfulness they identified as naturallyvarying individual differences. Four of the five facets of mindfulness were significantly associated with psychological distress in the predicted direction. (Observation of experience was unexpectedly positively associated with psychological distress.) Thus, even in the absence of an intervention intended to manipulate mindfulness, naturally-varying individual differences in the constructs identified by Baer et al. (2006) were associated with a meaningful mental health outcome, psychological distress. Despite this demonstrated relationship, the mechanism by which any of these facets of mindfulness might impact mental health remains unclear. 
Three possible mechanisms of action for which there is existing theoretical and empirical support are emotion regulation, non-attachment, and rumination. Emotion regulation refers to the ability to manage (negative) affect, typically by altering thoughts or behavior to address the source of distress or better cope with it. Mindfulness is hypothesized to improve the ability to manage negative affect by increasing familiarity with one's internal life, including satiety cues in binge eaters (Kristellar \& Hallett, 1999) and early signs of depressive relapse in those who have previously experienced a depressive episode (Teasdale, Segal, \& Williams, 1995). This information can then be used to cope with negative affect in effective ways (Shapiro et al., 2006). Brown \& Ryan (2003) proposed that future studies examine the impact of mindfulness on mood repair.

Buddhist texts also argue that mindfulness increases the ability to manage negative affect. Buddhist psychology posits that mindfulness facilitates insight into one's emotional life, which then enables one to liberate oneself from negative and destructive mental states (Ekman, Davidson, Ricard, \& Wallace, 2005). In Buddhist psychology, cognition and emotion are considered to be inextricable aspects of mental states (Goleman, 2003). Thus, the ability to liberate oneself from negative "mental states" is similar to the Western psychological concept of emotion regulation, in that the individual is better equipped to cope effectively with negative emotions. One of the only empirical studies to date on mindfulness' mechanisms of action supports its impact on emotion regulation. In an experimental study, participants who engaged in a mindful breathing exercise reported less negative affect and a greater willingness to view highly negative pictures than did participants in other experimental conditions (Arch \& Craske, 2006). 
A second potential mechanism, rumination, refers to repetitive, negative, and selffocused thoughts about the past or future (Trapnell \& Campbell, 1999). Ruminative thinking has been linked to depression and anxiety (Nolen-Hoeksema, 1991; Nolen-Hoeksema, 2000; Siegle, Moore, \& Thase, 2004). Mindful attention to the present moment, and the ability to control the focus of attention more broadly, are hypothesized to prevent one from becoming mired in ruminative thoughts (Baer, 2003; Teasdale et al., 1995). MBCT, a mindfulnessbased intervention for depression, uses this hypothesized relationship to prevent depressive relapse. MBCT assumes that individuals who have experienced a depressive episode associate transient dysphoric affect with depressive patterns of thinking, including feeling hopeless and self-devaluative. These thought patterns then amplify the dysphoria, creating a reciprocal spiral in which depressed affect and depressogenic thought patterns perpetuate each other. This pattern is believed to put individuals who have previously been depressed at increased risk for future depressive episodes. In MBCT, mindfulness is employed to assist individuals in viewing their thoughts and feelings as impermanent mental events. This attitude toward mental events is hypothesized to disrupt the connection between dysphoric affect and automatic, ruminative, depressogenic thought patterns, which short-circuits the depressogenic affect-cognition cycle the individual would otherwise experience (Teasdale et al., 1995). Thus, a previously depressed client might experience dysphoria, and begin to think in depressogenic ways. With mindfulness training, the individual notices the depressogenic cognitions but is less likely to believe that these thoughts are accurate representations of reality. The client is then less affected by the thoughts and is able to prevent escalating negative affect, preventing a depressive episode. 
For patients who have experienced three or more major depressive episodes, MBCT has been found to significantly reduce the risk of relapse over a 60 -week period, relative to treatment as usual (Teasdale et al., 2000). The specific mechanisms of action proposed by $\mathrm{MBCT}$, in which mindfulness reduces rumination, which then reduces depression, have not been tested. Consistent with this hypothesis, however, Jain et al. (2007) found that although mindfulness meditation and somatic relaxation were both associated with decreased selfreported psychological distress, only mindfulness meditation reduced rumination.

Furthermore, reductions in rumination mediated the impact of mindfulness meditation on psychological distress.

A third mechanism that may explain how mindfulness influences psychological distress is via its relationship with non-attachment. Attachments are objects or outcomes that people believe they must have to be happy (McIntosh, 1997). These include fleeting positive experiences, and avoidance of negative emotions (Dalai Lama \& Cutler, 1998; Hanh, 1998). Attachments are thought to cause suffering because they represent important goals, in the form of desired experiences or objects, that people believe they must have to be happy (McIntosh, 1997). People are most likely to ruminate when important goals are blocked (Martin \& Tesser, 1989); thus when attachments are unfulfilled, they often instigate rumination (McIntosh \& Martin, 1992).

The acceptance of present-moment experience associated with mindfulness may diminish this quality of resisting experience and imposing external situational requirements on one's happiness (Brown, Ryan, \& Creswell, 2007). Research indicates that individuals who report greater non-attachment are happier than those who report less non-attachment (McIntosh \& Martin, 1992). Furthermore, rumination has been found to mediate this 
relationship, such that when attachments are unfulfilled, people are more likely to ruminate, which then influences happiness (McIntosh \& Martin, 1992). Mindfulness has been hypothesized to be associated with greater non-attachment (Brown, Ryan, \& Creswell, 2007), which research suggests decreases rumination (McIntosh \& Martin, 1992), and which is in turn associated with less psychological distress (Jain et al., 2007). Thus, mindfulness may impact psychological distress through its influence on non-attachment, which then influences rumination and psychological distress. In summary, there is theoretical and some empirical support for the possibility that mindfulness influences psychological distress by increasing emotion regulation (which decreases psychological distress), increasing non-attachment (which decreases psychological distress by decreasing rumination), and decreasing rumination (which decreases psychological distress).

One way to explore potential causal mechanisms is to collect correlational data and test mediational models. In these models, a potential mechanism of action mediates the impact of one variable on another, such that a first variable influences a second (the mediator), which in turn influences a third. The first variable may or may not also directly influence the third variable. Although correlational data cannot demonstrate causation, the pattern of relationships in the data can be consistent with causal hypotheses. Structural equation models, which can examine both direct and indirect effects between variables, are well-suited to testing mediation.

Coffey and Hartman (2008) used structural equation modeling to explore possible mechanisms of action in the relationship between mindfulness and psychological distress. We found support for the role of emotion regulation, non-attachment, and rumination as possible mechanisms by which mindfulness might influence psychological distress. In 
particular, we found that mindfulness, as measured by the $\mathrm{MAAS}^{3}$ (which is most similar to the FFMQ subscale associated with acting with awareness instead of engaging in mindless, automatic behavior) was positively associated with the ability to manage negative affect, positively associated with non-attachment, or a tendency to view one's happiness as independent of external circumstances, and negatively associated with rumination. An improved ability to manage negative affect and less rumination in turn predicted decreased psychological distress. Non-attachment indirectly influenced psychological distress through its impact on rumination. Emotion regulation, non-attachment, and rumination were also associated with each other, such that an increased ability to manage negative affect was associated with a greater tendency to view happiness as independent of external circumstances and less rumination.

Although useful as a preliminary model of the mechanisms by which mindfulness might impact psychological distress, the Coffey and Hartman (2008) model utilized only the MAAS in measuring mindfulness. Thus, it considers only one of Baer et al.'s five mindfulness factors: specifically, the factor concerned with the degree to which one acts with awareness vs. functions on automatic pilot (Baer et al., 2006). The discovery that current mindfulness measures encompass five correlated constructs raises the possibility that different aspects of what is considered "mindfulness" may influence each other and psychological distress in different ways. For example, some aspects of mindfulness measured by the FFMQ, such as observation of experience, may precede other aspects of mindfulness, such as the ability to describe one's experience.

\footnotetext{
${ }^{3}$ The FFMQ was not yet available when this study was conducted.
} 
Were some aspects of mindfulness to precede or facilitate the development of other aspects of mindfulness, the boundary between constructs that are considered "mindfulness" and those that are mindfulness' sequelae or mechanisms of action would become unclear. It may become more useful to discuss mindfulness in terms of its component processes (e.g., observation of experience, describing experience, etc.) than to summarize these processes with a single term (i.e., mindfulness). For example, present-centered attention and an accepting or nonjudging attitude towards one's experience are common to most mindfulness definitions (e.g., Kabat-Zinn, 1994; Bishop et al., 2004; Linehan, 1993). It is possible that these core components of mindfulness facilitate some of the less commonly-included mindfulness components (e.g., the ability to describe experience or a non-reactive stance towards internal experience). The facilitative relationship between different components might explain the presence of some less commonly-included components in mindfulness measures (e.g., describing experience or a non-reactive stance towards internal experience): although these facets of mindfulness may not be integral to the construct, they may be likely to co-occur with integral facets (e.g., present-centered attention and a nonjudgmental orientation towards experience).

Furthermore, some of the components of mindfulness assessed may influence psychological distress only indirectly, through their influence on other variables such as emotion regulation or rumination. In contrast, other aspects of mindfulness may exert both direct and indirect influences on psychological distress. Thus, the term mindfulness appears to encompass a heterogeneous set of related constructs whose relationships with each other and with psychological distress are not fully known. For these reasons, a revised model for 
mindfulness' mechanisms of action that accounts for the multi-faceted nature of the construct is needed.

The model Coffey and Hartman (2008) proposed may also over-simplify the process of emotion regulation. Their model relied on only a single measure for emotion regulation, the Repair subscale from the Trait Meta-Mood Scale (TMMS; Salovey, Mayer, Goldman, Turvey, \& Palfai, 1995). This measure examines the ability to repair negative moods using cognitively-oriented techniques such as thinking positive thoughts. Other researchers have suggested that emotion regulation may entail six different abilities (Gratz \& Roemer, 2004). Difficulties with emotion regulation may reflect disruptions in any or all of these six abilities, including: nonacceptance of emotional experience; lack of clarity about one's feelings; lack of awareness of one's emotions; difficulty engaging in goal-directed behavior; impulse control difficulties; and limited access to emotion regulation strategies. Nonacceptance of emotional experience assesses judgmental and punitive reactions to the respondent's own distress. Lack of clarity assesses difficulty identifying what one is feeling, while awareness of feelings assesses attentiveness to and acknowledgement of one's emotional experience. Difficulties engaging in goal-directed behavior measures a tendency to decompensate in the presence of negative affect, while impulse control difficulties measures a tendency to engage in impulse, maladaptive behavior in the presence of negative affect. Lastly, limited access to emotion regulation strategies assesses a tendency to feel hopeless and disempowered to improve psychological distress. The Difficulties in Emotion Regulation Scale (DERS; Gratz \& Roemer, 2004) measures this comprehensive and integrative set of abilities related to emotion regulation. A revised model for mindfulness' mechanisms of action should reflect the multiple distinct processes that are collectively referred to as emotion regulation. Some 
facets of emotion regulation, such as acceptance of emotional experience, may precede other aspects, such as the ability to engage in goal-directed behavior despite the presence of negative affect. Similarly, awareness of one's emotions may precede clarity in identifying an emotion.

The task of constructing a revised model that incorporates the multi-faceted nature of emotion regulation is complicated by the unknown relationship between the different aspects of mindfulness, as measured by the FFMQ, and the different aspects of emotion regulation, as measured by the DERS and TMMS. Certain aspects of emotion dysregulation, such as nonacceptance of emotional response, appear intimately related to aspects of mindfulness, such as non-judging of one's experience. Furthermore, both the mindfulness and the emotion regulation research literatures draw heavily on Marsha Linehan's (1993) seminal work on treatment of borderline personality disorder. In identifying the importance to emotion regulation of attending to, accepting, and recognizing emotional responses, Gratz and Roemer (2004) reference Linehan (1993), as do Baer et al. (2004) when assembling items for mindfulness subscales on the KIMS. If, as Linehan (1993) suggests, mindfulness is important in facilitating emotion regulation, and if these two constructs are also composed of multiple smaller processes, it is possible that the boundary between them has become blurred in the attempt to model both constructs as comprehensively as possible. Thus, as part of determining how aspects of mindfulness impact more proximal variables, which then influence psychological distress, it is also necessary to explore the overlaps among these constructs. 


\section{The Current Studies}

In summary, research indicates that higher levels of most aspects of dispositional mindfulness are associated with less psychological distress, even when mindfulness is examined as a naturally-varying individual difference in participants with little or no formal mindfulness training. There is theoretical and empirical support for the possibility that mindfulness might impact psychological distress by improving the ability to manage negative affect, increasing a sense that one's happiness is independent of external circumstances, and decreasing rumination, which are in turn associated with psychological distress. Recent work on aspects of mindfulness (Baer et al., 2006) and emotion regulation (Gratz \& Roemer, 2004) indicates that these constructs may be complex and multi-faceted, however. The relationships among different facets of mindfulness and different facets of emotion regulation are not wellunderstood.

The present sequence of two studies builds on these findings to explore how different facets of dispositional, naturally-varying mindfulness might influence each other and mental health. The first study explored the relationship between current conceptualizations of mindfulness and emotion regulation. In this study, the appropriateness of treating current mindfulness and emotion regulation measures (i.e., the FFMQ, DERS, and subscales from the TMMS) as reflecting two separate and distinct constructs was explored. A series of exploratory factor analyses were conducted to better understand the constructs that are captured by these measures. The final solution was then tested in a confirmatory factor analysis, and the possibility that some of the constructs identified might precede or facilitate others was tested via a path analysis. The second study tested a more complex model of how the constructs identified in Study 1 might influence each other, the mediators proposed by 
Coffey and Hartman (2008), and two aspects of mental health, psychological distress and "flourishing" mental health, or a sense that one is living a rich and satisfying life.

These two studies tested several hypotheses. The first study was designed to better understand mindfulness, as a construct. It explored the hypotheses that 1) multiple distinct processes are subsumed under the terms "mindfulness" and "emotion regulation;" 2) some of the same processes are simultaneously considered "mindfulness" and "emotion regulation;" and 3) some of these processes may facilitate or enhance others, suggesting that they are not simply co-occurring but may be causally related.

The second study explored possible mechanisms of action by which mindfulness might influence mental health. It tested the hypothesis the aspects of mindfulness identified in Study 1 influenced mental health by improving the ability to regulate negative affect, decreasing a sense of reliance on external circumstances for one's happiness, and decreasing rumination, just as they did in the model tested by Coffey and Hartman (2008). In addition, this study tested the possibility that mindfulness might beneficially impact two different aspects of mental health - psychological distress and flourishing - via the same mechanisms. 


\section{STUDY 1}

Methods

\section{Participants}

A total of 399 undergraduate students (60\% female) from the University of North Carolina at Chapel Hill participated in this study in exchange for credit towards a course requirement. Participants completed the study via online questionnaires during the thirteenth of 16 weeks in the Spring 2006 semester. The sample ranged in age from 18 years to 24 years $(M=19.2$

years, $S D=1.11$ years). Sixty percent of the sample were first-year students. ${ }^{4}$ Approximately $85.2 \%$ of the sample (340 participants) indicated that they had either never meditated (143 participants), had not meditated in the past 6 months (139 participants), or had done so less than once/month during the previous six months (58 participants). An additional $6.5 \%$ of the sample indicated that they had meditated an average of 1-3 times/month during the previous six months. The remaining $8.3 \%$ of the sample (33 participants) indicated that they had meditated an average of once/week or more often during the previous six months.

\section{Materials}

Five-Factor Mindfulness Questionnaire (FFMQ; Baer et al., 2006). The FFMQ is a 39-item self-report measure that examines five hypothesized components of mindfulness. These five subscales include observation of one's internal experience and sensations (FFMQ Observe), non-judging of experience (FFMQ Nonjudging); describing one's experience 
(FFMQ Describe); nonreactivity to inner experience (FFMQ Nonreactivity), and acting with awareness versus on automatic pilot (FFMQ Acting with Awareness). Items include: "I pay attention to sensations, such as the wind in my hair or sun on my face" (FFMQ Observe); "I tell myself I shouldn't be feeling the way I'm feeling" (FFMQ Nonjudging); "I have trouble thinking of the right words to express how I feel about things" (FFMQ Describe); "I perceive my feelings and emotions without having to react to them" (FFMQ Nonreactivity); and "I don't pay attention to what I'm doing because I'm daydreaming, worrying, or otherwise distracted" (Acting with awareness subscale, reverse-scored). Respondents answer on a 5point Likert scale from 1 (never or very rarely true) to 5 (very often or always true). In this study, all items from the Acting with Awareness vs. on Automatic Pilot subscale were scored so that Acting with Awareness responses produced higher scores, indicating that high scores on the subscale reflected Acting with Awareness rather than its opposite, Automatic Pilot. For this reason, this subscale is referred to as "Acting with Awareness" throughout the rest of this document. Internal consistency reliability (Cronbach's $\alpha$ coefficient) for the FFMQ subscales in this sample were: FFMQ Nonreactivity $=.72$; FFMQ Observe $=.74$; FFMQ Acting with Awareness $=.85$, FFMQ Describe $=.86$, and FFMQ Nonjudging $=.87$.

Difficulties in Emotion Regulation Scale (DERS, Gratz \& Roemer, 2004). The DERS is a 36-item self-report questionnaire that was designed to assess six aspects of emotional dysregulation. To facilitate data analysis and interpretation in this study, all DERS subscales were reverse-scored to reflect an absence of emotion regulation difficulties, or the presence of emotion regulation ability. Subscales include emotional awareness (DERS Awareness), acceptance of emotional responses (DERS Acceptance), emotional clarity (DERS Clarity),

\footnotetext{
${ }^{4}$ Information on racial/ethnic background was not collected.
} 
ability to in engage in goal-directed behaviors (DERS Goals), impulse control (DERS Impulse control), and access to emotion regulation strategies (DERS Strategies). Questions include: "When I'm upset, I acknowledge my emotions" (DERS Awareness); "When I'm upset, I feel guilty for feeling that way" (DERS Acceptance); "When I'm upset, I can still get things done" (DERS Goals); "When I'm upset, I lose control over my behaviors," (DERS Impulse control); and "When I'm upset, I believe there is nothing I can do to make myself feel better" (DERS Strategies). Participants respond along a 5-point Likert scale where 1 represents "almost never" and 5 represents "almost always."). Internal consistency reliability (Cronbach's $\alpha$ coefficient) for the DERS subscales in this sample were: DERS Acceptance $=$ $.90 ;$ DERS Goals $=.87$, DERS Impulse control $=.87$, DERS Awareness $=.76$, DERS Strategies $=.87$, and DERS Clarity $=.81$.

Trait Meta-Mood Scale (TMMS; Salovey et al., 1995). The TMMS contains subscales that measure the ability to discriminate clearly among moods (TMMS Clarity) and regulate moods (TMMS Repair). The Clarity subscale consists of 11 items with statements such as, "I am usually very clear about my feelings," whereas the Repair subscale includes 6 items with statements such as, "When I become upset, I remind myself of the pleasures in life." Participants respond along a five-point scale with ratings from $1=$ strongly disagree to $5=$ strongly agree. Internal consistency reliabilities (Cronbach's $\alpha$ coefficient) in this sample were 0.80 for the Repair subscale and .85 for the Clarity subscale.

\section{$\underline{\text { Results }}$}

\section{Overview of data analytic strategy.}

Data analysis entailed four steps. The first step tested the appropriateness of treating existing mindfulness and emotion regulation measures as separate and distinct constructs. 
These analyses suggested that these terms reflected heterogeneous constructs. The second step consisted of a series of exploratory factor analyses to investigate the presence of common factors in existing mindfulness and emotion regulation measures. The third step subjected the common factors identified in the exploratory factor analyses to a more rigorous test in a confirmatory factor analysis. The last step tested a theory-driven model for the potential relationships among the common factors.

The following models were tested using LISREL 8.51 (Jöreskog \& Sörbom, 2001) and Full Information Maximum Likelihood (FIML) estimation. This estimation procedure assumes that all indicators are normally distributed, thus the data were first examined for normality. To reduce the effects of negative skew, the DERS Acceptance, DERS Impulse control, DERS Strategies, DERS Clarity, and the TMMS Repair subscales were transformed by squaring them, resulting in the following distributions: DERS Acceptance (skewness = 0.30 , kurtosis $=-0.54)$; DERS Clarity ( (skewness $=0.02$, kurtosis $=-0.47)$; DERS Impulse control $($ skewness $=-0.59$, kurtosis $=$ 0.60 ); and DERS Strategies (skewness $=-0.26$, kurtosis $=-0.87$ ).

After transformation, scatterplots representing the relationship between each set of indicators in these analyses (a total of 91 scatterplots) were created to examine potential outliers. Although isolated scatterplots suggested different potential outliers, no participant produced consistently unusual patterns of scores. For this reason, no participants were excluded as outliers and the final sample consisted of 399 participants.

T-tests examining the impact of gender on all variables included in the model unexpectedly revealed more significant tests than would be expected on the basis of chance. In particular, woman scored higher on the DERS Awareness of emotions subscale, whereas 
men scored higher on DERS Clarity, FFMQ Nonreactivity, and DERS Strategies. ${ }^{5}$ Analysis of variance tests examining the impact of Year in School on all variables in the model revealed only one significant difference, which might be expected on the basis of chance. ${ }^{6}$ Age also predicted only one variable, which might also be expected on the basis of chance. ${ }^{7}$ Indicator descriptive statistics and inter-correlations are presented in Table 1.

\section{Step 1: Confirmatory Factor Analysis for Original Mindfulness and Emotion Regulation Scales.}

As described above, Study 1 consisted of four sets of analyses. The first step consisted of a confirmatory factor analysis which examined whether subscales from a questionnaire designed to measure mindfulness load exclusively on a Mindfulness factor, whereas subscales from a questionnaire designed to measure emotion regulation load exclusively on an Emotion Regulation factor. This analysis included the TMMS Clarity scale, which we hypothesized would load on the Mindfulness factor, and the TMMS Repair scale, which we hypothesized would load on the Emotion Regulation factor. This model proved to be a very poor fit to the data. It produced a RMSEA of $0.16\left(\mathrm{CI}_{90}=0.15-0.17\right.$; $\left.\chi^{2}=743.64, d f=64, p<0.0001\right)$. Approximately $0.04 \%$ of the data were missing. ${ }^{8}$ Excluding

\footnotetext{
${ }^{5}$ This pattern does not correspond to any pre-existing theory or expectations about gender differences. Only one of these significant tests was replicated in the second sample (below).

${ }^{6}$ On the DERS Impulse control subscale, first-year students and seniors scored higher than sophomores, who scored higher than juniors. To the author's knowledge, this pattern of means is not consistent with any preexisting theory about developmental differences, nor was it replicated in the second sample (below).

${ }^{7}$ Age also significantly predicted Impulse control, which is unsurprising given the close relationship between Year in School and Age. The pattern of means produced for Age was U-shaped and followed the same pattern as did the relationship between Impulse control and Year in School.

${ }^{8}$ The minimum discrepancy function, which is required for fit statistics such as the Non-normed Fit Index (NNFI, also known as the Tucker-Lewis Index) and the Comparative Fit Index (CFI), is not defined when there are missing data in a sample, thus these fit statistics are not available.
} 
the two TMMS subscales, and testing the model with only the subscales from the FFMQ and DERS, produced a model that LISREL could not estimate.

\section{Step 2: Exploratory Factor Analyses.}

The preceding analysis indicated that current measures for mindfulness and emotion regulation were not well-modeled by separate mindfulness and emotion regulation factors. A second set of analyses then explored whether common factors might still be present in the data. This set of analyses was conducted using SPSS.

All five FFMQ subscales, all six DERS subscales, and the two TMMS subscales were entered in an exploratory factor analysis using maximum likelihood estimation and direct oblimin rotation. Both the eigenvalues and the scree plot graph of the eigenvalues suggested a three or four factor solution. The three-factor solution produced poor model fit and an unclear factor structure, with the DERS Acceptance subscale loading weakly on two factors and the FFMQ Observe subscale failing to load on any factor $\left(\chi^{2}=168.06, d f=42, p<0.0001\right.$; Table 2). Extracting four factors, rather than three, improved the model fit and the interpretability of the factors $\left(\chi^{2}=80.42, d f=32, p<0.0001\right.$; Table 3$)$. In particular, as depicted in table 3, the following subscales now clearly loaded together: Factor 1 consisted of the DERS Awareness subscale and a weaker factor loading by the FFMQ Observe subscale $(\lambda=0.37)$; Factor $2=$ FFMQ Nonreactivity, TMMS Repair, DERS Goals, DERS Impulse control, and DERS Strategies; Factor 3 = TMMS Clarity, DERS Lack of Clarity, and the FFMQ Describe; and Factor 4 = FFMQ Nonjudging and DERS Acceptance. Factor loadings of 0.36 or higher were considered to indicate clear and meaningful factor loadings. Using this standard, the FFMQ Acting with Awareness subscale did not load meaningfully 
on any factor and was excluded from subsequent attempts to identify common factors in the data.

A third exploratory factor analysis was then conducted with all preceding indicators except the FFMQ Acting with Awareness subscale, to ensure that the pattern of factor loadings was stable and not disrupted by exclusion of this measure. Results from this analysis confirmed the stability of the pattern of factor loadings $\left(\chi^{2}=44.43, d f=24, p=0.007\right.$; Table 4).

\section{Step 3: Confirmatory Factor Analyses for the Four Factors Identified in the} Exploratory Factor Analyses.

A confirmatory factor analysis was then conducted to test the model suggested by the exploratory factor analyses, which consisted of four common factors and their indicators. This model is depicted in Figure 1. Although the fit statistics for this model suggested a reasonable fit for the data, the output provided two indications that the fit statistics did not reflect true model fit. First, the solution is a "Heywood case," with an estimated unique variance of -1.67 for the DERS Awareness subscale and a reported squared multiple correlation (SMC) for that variable of 2.67. The second indication was the standardized fitted residuals for the common factor comprised of the DERS Awareness and FFMQ Observe subscales. Standardized fitted residuals are the residuals produced when a model is fit to the data. Absolute values larger than 1.96 are statistically significant. Although some significant residuals might be expected on the basis of chance, particularly given the presence of 78 correlations in the model, four of the six significant standardized fitted residuals were associated with either the DERS Awareness or FFMQ Observe subscales. For these reasons, although the overall model fit statistics for the four-factor model depicted in Figure 1 were 
acceptable, the common factor uniting the DERS Awareness and FFMQ Observe subscales appeared problematic.

For this reason, a second confirmatory factor analysis was conducted. This model was identical to the previous one, but omitted the DERS Awareness, the FFMQ Observe, and their common factor (Figure 2). This model produced an acceptable fit, with a RMSEA of $0.055\left(\mathrm{CI}_{90}=0.038-0.072 ; \chi^{2}=70.52, d f=32, p=0.0001\right)$. All SMCs, unique variances, and residuals also suggested that the model was a good fit for the data. The factor intercorrelations are included in Table 5.

Examination of the subscales associated with each of the three factors identified in this analysis suggests that they can be described as acceptance of emotional experience, clarity about one's feelings, and the ability to control behavior in the presence of negative affect. The FFMQ Nonjudging and DERS Acceptance subscales associated with the Acceptance factor ${ }^{9}$ feature items that address judgmental and self-critical responses to unpleasant internal experiences. The Clarity factor consists of the FFMQ subscale for the ability to describe one's experience (FFMQ Describe), the TMMS subscale for clarity about one's experience (TMMS Clarity), and the DERS subscale for clarity about one's experience (DERS Clarity). Interestingly, the composition of this factor suggests that recognition of one's internal experience (e.g., sadness) is isometric with the ability to label internal experience (e.g., "I am sad").

The third factor consists of subscales from the FFMQ, the TMMS, and the DERS. These subscales measure the ability to notice internal distress without having to behaviorally react to it (FFMQ Nonreactivity), the ability to alter negative moods (TMMS Repair), the 
ability to perform necessary, goal-focused behavior in the presence of negative affect (DERS Goals), the ability to control behavior in the presence of negative affect (DERS Impulse), and access to emotion regulation strategies in the presence of negative affect (DERS Strategies). This factor appears to be behaviorally-oriented and examination of the questionnaires items for each of the subscales suggests that this factor is most closely linked to traditional conceptions of emotion regulation, which often involve behavioral strategies for managing negative affect (as opposed to noticing the affect or not judging the affect). This factor also includes three of the six DERS subscales in the factor analysis. For these reasons, the factor associated with the ability to control behavior in the presence of unpleasant internal experience will be referred to as "Negative emotion regulation." The word "negative" makes explicit the fact that this work investigates the way negative, as opposed to positive, emotions are regulated.

Step 4: Path Analysis Examining the Relationships among the Common Factors.

Steps 1-3 in the preceding analyses revealed that the terms "mindfulness" and "emotion regulation," as commonly used and measured, encompass an overlapping and heterogeneous set of constructs. This presents a dilemma in the attempt to understand mindfulness: which of these constructs is mindfulness? This question becomes especially complicated because each factor in the preceding factor analysis featured subscales from the FFMQ, which was designed to measure mindfulness. Moreover, the two remaining FFMQ subscales, which did not load on any of the three common factors, may still represent important aspects of mindfulness.

\footnotetext{
${ }^{9}$ Factor names are capitalized throughout this document to distinguish the statistically-measurable role of
} 
Theoretical work by Bishop et al. (2004) suggests that a pure rendering of mindfulness, one which attempts to distill mindfulness from its sequelae, might consist of two factors: one involving present-focused attention and a second which involves acceptance of the object of one's attention. In the current data set, the acceptance portion of the Bishop et al. (2004) definition could be represented with the Acceptance factor, consisting of the FFMQ Nonjudging and DERS Acceptance subscales. The attention portion of this definition should optimally be represented by measures that assess present-focused attention, such as the FFMQ Observe or the DERS Awareness. Step 3 in the preceding analyses indicated that only one of these measures should be used as an indicator for present-centered attention, because they are not sufficiently similar to be considered expressions of the same common factor. Examination of the individual questions for each measure revealed that the DERS Awareness items specifically address attention to one's emotions, whereas the FFMQ Observe subscale addresses attention to present-moment experience more broadly, including physical sensations and the external environment. The latter was considered to more accurately reflect what is meant by present-centered attention. Thus, the attentional component of the Bishop et al. (2004) operational definition could be represented in the present data set by permitting the FFMQ Observe subscale to load on its own "Attention" factor.

The last step in this sequence of analyses explored the possibility that some of the constructs identified in the preceding analyses might be sequelae of mindfulness, as operationally defined by Bishop et al. (2004). If the pattern in the data supported this possibility, it would suggest that some constructs that are generally subsumed under the term 
"mindfulness" are, in fact, consequences of mindfulness. Interestingly, dialectical behavior therapy (DBT) teaches skills related to each of the common factors identified in these analyses. In DBT, participants are taught to pay attention to their experience and to notice judgments about their experience as a way of beginning to develop some clarity about their feelings and subsequently engage in more effective regulation of negative affect (Linehan, 1993). Thus, the last step in the analyses tested a theory-driven model in which mindfulness, as represented in the model by the Attention and Acceptance factors, predicted Clarity about one's emotional experience, which in turn predicted Negative Emotion Regulation (Figure 3). The model also examined the possible direct effects that Attention and Acceptance might exert on Negative Emotion Regulation.

This model did not include the FFMQ Acting with Awareness subscale, even though this subscale most closely reflects how mindfulness was measured in a previous model for mindfulness' mechanisms of action (Coffey \& Hartman, in press). Although engaging in mindful behavior appeared to be broadly related to multiple aspects of the model, it did not clearly fit at any specific juncture in the proposed processes. Several other (non-theorydriven) models, which included the FFMQ Acting with Awareness subscale, were tested as part of these analyses but none of them fit the data as well as did the original model. More detail about these models is included in Appendix A.

The model produced an acceptable fit to the data with an RMSEA of $0.059\left(\mathrm{CI}_{90}=\right.$ $\left.0.044-0.075 ; \chi^{2}=93.84, d f=39, p<0.0001\right)$. In this model, both the Attention and Acceptance factors significantly predicted Clarity and Emotion regulation. This finding is noteworthy for two reasons. First, Brown and Ryan (2004) found that their "Attention" factor, which actually measured "acting with awareness" behavior using Baer et al.'s 2006 
terminology, subsumed an attitude of acceptance. Results from this study suggest that present-centered attention, as measured by the FFMQ Observe subscale, does not subsume an attitude of acceptance, because the Acceptance factor independently affected Clarity and Emotion regulation, above and beyond the influence of the Attention factor.

Second, Baer et al. (2004) and Baer et al. (2006) found that their Observe subscale (which was the single indicator for the Attention factor) was inconsistently related, or related in the non-predicted direction, to other relevant constructs. In this data set, Attention was significantly related to Clarity and Emotion regulation in the predicted directions. It was not, however, significantly correlated with the Acceptance factor $(r=-0.08, z=-1.12, p=0.26)$, which is consistent with work by Baer et al. (2006), and differs from Baer, Smith, and Allen's (2004) finding that these two constructs were negatively correlated. Although the subsample of frequent meditators $(n=33)$ is not sufficient to test the proposed model for meditators alone, it should also be noted that the correlation between the FFMQ Observe subscale and the FFMQ Nonjudging subscale, which Baer et al. (2006) proposed may differ in those with and without meditation experience, was significantly negative at $r=-0.16$ for the 366 participants who reported that they did not meditate regularly, and not significant at $r$ $=0.01$ for the 33 participants in the full sample who reported meditating once/week or more often.

As depicted in Figure 3, Attention to one's experience and Acceptance of that experience jointly influenced an individual's Clarity about his or her experience. Clarity about one's experience partially mediated the impact of both Attention and Acceptance on one's ability to effectively regulate negative affect. The model explained $43 \%$ of the variance in Clarity and $50 \%$ of the variance in Negative Emotion Regulation. 


\section{Brief Discussion of Study 1.}

Each of the three study hypotheses was confirmed. First, the results are consistent with the hypothesis that multiple, conceptually-distinct processes are subsumed under the terms "mindfulness" and "emotion regulation." A confirmatory factor analysis tested the appropriateness of modeling current conceptualizations of these terms as two distinct constructs. This model fit poorly, indicating that current conceptions of both mindfulness and emotion regulation encompass a heterogeneous set of processes.

Second, results supported the hypothesis that some of the same processes are simultaneously considered mindfulness and emotion regulation. Exploratory factor analyses indicated the presence of important areas of overlap between current conceptualizations of mindfulness and emotion regulation. Examination of these areas of overlap revealed that these constructs may be more accurately thought of as acceptance of internal experience, recognition of internal experience, and the ability to control behavior in the presence of unpleasant internal experiences. The three common factors were significantly correlated with each other. Notably, each of the common factors identified in these analyses included at least one subscale from the mindfulness questionnaire used here and at least one subscale from the emotion regulation questionnaire used here, demonstrating the conceptual commingling of mindfulness and emotion regulation in existing measures for these constructs. Two aspects of current conceptualizations of mindfulness, specifically present-centered attention and acting with awareness, were unique in that they were not isometric with any aspects of emotion regulation. Interestingly, acting with awareness and present-centered attention were also not significantly correlated with each other. 
This study then examined the relationships among the different constructs identified in the preceding analyses. Specifically, this study tested a third hypothesis that these constructs may be related because they contribute to or facilitate the expression of each other. A path analysis supported this hypothesis. Both present-centered attention and acceptance of internal experience, collectively recognized as "mindfulness" according to the Bishop et al. (2004) operational definition of this construct, contributed to clarity about one's internal experience, which in turn contributed to the ability to manage negative affect. In other words, a greater tendency to attend to one's present-moment experience and increased acceptance of one's experience were associated with a greater ability to identify one's emotions. Higher levels of this ability were in turn associated with an improved ability to manage emotions when they were negative. The two aspects of mindfulness were not significantly associated with each other. 


\section{STUDY 2}

Methods.

Participants.

A total of 413 undergraduate students (71\% female) from the University of North Carolina at Chapel Hill participated in this study in exchange for credit towards a course requirement. Data were collected via online questionnaires during the third of 15 weeks in the Fall 2007 semester. The sample ranged in age from 18 years to 23 years $(M=18.7$ years, $S D=0.95$ years). Fifty-seven percent of the sample were first-year students. Approximately $94 \%$ of the sample (388 participants) indicated that they had either never meditated or had done so less than once/month during the previous six months. An additional $2.7 \%$ of the sample indicated that they had meditated an average of 1-3 times/month during the previous six months. The remaining $3.7 \%$ of the sample (14 participants) indicated that they had meditated an average of once/week or more often during the previous six months.

\section{Materials.}

Five-Factor Mindfulness Questionnaire (FFMQ; Baer et al., 2006). Described above. Internal consistency reliabilities (Cronbach's $\alpha$ coefficients) for the FFMQ subscales in this sample were: FFMQ Nonreactivity $=.71$; FFMQ Observe $=.71$; FFMQ Acting with Awareness $=.86 ;$ FFMQ Describe $=.88 ;$ and FFMQ Nonjudging $=.88$.

Difficulties in Emotion Regulation Scale (DERS, Gratz \& Roemer, 2004). Described above. Internal consistency reliabilities (Cronbach's $\alpha$ coefficients) for the DERS subscales 
in this sample were: DERS Acceptance $=.87$; DERS Goals $=.91$, DERS Impulse control $=$ $.88 ;$ DERS Awareness $=.79 ;$ DERS Strategies $=.88 ;$ and DERS Clarity $=.85$

Trait Meta-Mood Scale (TMMS; Salovey et al., 1995). Described above. Internal consistency reliabilities (Cronbach's $\alpha$ coefficients) in this sample were 0.82 for the Repair subscale and .87 for the Clarity subscale.

The Linking Inventory (McIntosh \& Martin, 1992). The Linking Inventory measures the extent to which subjects believe their happiness is independent of obtaining positive outcomes. It consists of 22 dichotomous, forced-choice questions, used to obtain participants' judgments about the way specific outcomes affect their happiness. For example, one item states, "One day you realize that you have all the things you want -- the job you want, the spouse you want, the free time you want." The response options for this item are "(a) This will not directly influence how happy I am, because happiness is something I determine, regardless of what happens to me," and "(b) If I have all the things I want, then I will be very happy." In this example, Response B represents a linking orientation, while Response A represents a nonlinking, or non-attached, orientation. Internal consistency reliability (Cronbach's $\alpha$ coefficient) for the Linking Inventory was 0.77 in this sample.

Rumination. The Rumination subscale on the Rumination-Reflection Questionnaire (RRQ; Trapnell \& Campbell, 1999) measures "ruminative self-attention," or the tendency to dwell on, rehash, or reevaluate events and experiences. It consists of 12 items, which include statements such as "Long after an argument or disagreement is over, my thoughts keep going back to what happened." Participants respond along a five-point Likert scale, with response options ranging from "strongly disagree" to "strongly agree." The Rumination subscale has a 
reported alpha coefficient of .90 (Trapnell \& Campbell, 1999). Internal consistency reliability (Cronbach's $\alpha$ coefficient) for the RRQ Rumination scale was 0.90 in this sample.

Psychological Distress. The Brief Symptom Inventory (BSI; Derogatis, 1983) is a 53item Likert-scale derived from the Hopkins Symptom Checklist (SCL-90-R; Derogatis, 1977). The BSI produces multiple indices of distress: the depression (DEP) and anxiety (ANX) subscales were used in this study. Internal consistency reliabilities (Cronbach's $\alpha$ coefficients) in this sample were as follows: BSI Depression $=0.82$; BSI Anxiety $=0.74$.

Satisfaction with Life Scale (SWLS; Diener, Emmons, Larsen, \& Griffin, 1985). The SWLS is a 5-item scale that assesses participants' global satisfaction with their lives and circumstances. Participants indicate agreement on a 7-point scale with each item, including "So far I have gotten the important things I want in life." Internal consistency reliability (Cronbach's $\alpha$ coefficient) in this sample was 0.86 .

Short flourishing (Keyes, 2005). The Short flourishing measure is a 14-item measure that assesses several aspects of hedonic and eudaimonic well-being, including relationships with others and society at large, environmental mastery, self-acceptance, purpose in life, personal growth, and autonomy. Respondents rate the frequency with which they felt various aspects of eudaimonic well-being in the past month on a 6-point scale from "never" to "every day”. Responses were summed to create a total score for the measure. Internal consistency reliability (Cronbach's $\alpha$ coefficient) in this sample was 0.90 .

\section{$\underline{\text { Results }}$}

Overview of data analytic strategy.

Analyses for Study 2 consisted of first replicating the factor structure from Study 1 in an independent sample, to ensure that the factor structure was not a result of unique sample 
characteristics in Study 1. The second part of the analyses involved integrating results from Study 1 on the nature of mindfulness and emotion regulation with the potential mechanisms of action Coffey and Hartman (2008) explored in their original model. This included exploring potential mechanisms of action in the relationship between mindfulness and psychological distress, and between mindfulness and flourishing mental health.

Models were tested using LISREL 8.51 (Jöreskog \& Sörbom, 2001) and Full Information Maximum Likelihood (FIML) estimation. This estimation procedure assumes that all indicators are normally distributed, thus the data were first examined for normality. Distributions of scores for the DERS Acceptance, DERS Clarity, TMMS Repair, DERS Strategies, and DERS Impulse control measures were more negatively skewed in this sample than for Study 1, perhaps reflecting either improved functioning at the beginning of the semester or personality characteristics associated with participants who complete course requirements at the beginning of the semester. To reduce the effects of negative skew, the DERS Acceptance, DERS Clarity, TMMS Repair, and DERS Strategies were transformed by squaring them, resulting in the following distributions: DERS Acceptance (skewness $=-0.50$, kurtosis $=-0.37)$; DERS Clarity (skewness $=-0.32$, kurtosis $=-0.27$ ); TMMS Repair (skewness $=-0.27$, kurtosis $=-0.37)$; and DERS Strategies (skewness $=-0.58$, kurtosis $=-$ 0.32). The DERS Impulse control was highly negatively skewed, indicating that most respondents felt very competent to control their impulses. This variable was transformed by squaring it in Study 1; in Study 2, taking this variable's cube produced a more normal distribution (skewness $=-0.73$, kurtosis $=-0.43$ ). To reduce the effects of positive skew, the BSI Depression and BSI Anxiety scales were transformed by taking their square root, resulting in the following distributions: BSI Depression (skew $=0.80$, kurtosis $=0.17)$ and 
BSI Anxiety $(\mathrm{skew}=0.79$, kurtosis $=0.87)$. Indicator means, standard deviations and intercorrelations are presented in Table 6.

T-tests by gender on all variables included in the model revealed only one significant difference, which does not differ from the number expected by chance (men scored higher on the FFMQ Nonreactivity subscale). Neither year in school nor age significantly predicted any of the variables in the model.

\section{Step 1: Confirmation of Factor Structure from Study 1.}

A confirmatory factor analysis tested the factor structure featured in Figure 2 to ensure that it appropriately modeled the relationships among the measures in a new and independent sample. Results from this analysis indicated that the model was an acceptable fit

for the data $\left(\mathrm{RMSEA}=0.066 ; \mathrm{CI}_{90}=0.051-0.083 ; \chi^{2}=90.34, d f=32, p<0.0001\right)$. Standardized factor loadings and factor inter-correlations for this model are included in Tables 7 and 8, respectively. These are similar to the factor inter-correlations and factor loadings presented in Table 5 and Figure 2, reflecting the stability of these estimates across samples.

Step 2: Mechanisms of Action in the Relationship between Mindfulness and Psychological Distress

The second step in these analyses united the factor structure developed in Study 1 (and confirmed in Step 1 of Study 2) with the model Coffey and Hartman (2008) proposed and tested for mindfulness' mechanisms of action. In the first part of this combined model, mindfulness, as operationally defined as Attention to present-moment experience and Acceptance of one's internal experience, contributes to Clarity about one's internal 
experience. Clarity, in turn, facilitates regulation of negative affect. In the second part of this model, an increased ability to regulate negative affect contributes to greater non-attachment, which is then associated with less rumination. Improved negative emotion regulation and decreased rumination then predict less psychological distress.

Three of the factors in the model (i.e., Attention, Non-attachment, and Rumination) were each measured with only one questionnaire/factor. Therefore, these factors did not have a sufficient number of indicators to produce measurement error estimates. Failure to account for measurement error and unreliability in a variable can underestimate the variable's influence on other variables in the model. The Rumination measure (i.e., the RRQ Rumination) had high internal reliability (unlike the measures for Attention and Nonattachment), and a sufficient number of items to split the scale. Thus, this measure was divided into item parcels, such that the first half of the scale was treated as one indicator for the factor while the second half of the scale was treated as a second indicator for the factor. This allowed computation of measurement error for the Rumination factor and inserted additional degrees of freedom into the model, creating a more rigorous test of the model while preserving degrees of freedom appropriate to the sample size. For the other two factors measured with one variable, Attention and Non-attachment, calculations based on Cronbach's $\alpha$ coefficient were entered into the model to approximate measurement error for the factor.

Given the absence of previous empirical work exploring the relationships among these constructs, coefficients for all possible paths were freed for estimation in a first version of the model. This all-inclusive model produced an acceptable fit, with a RMSEA of 0.068 $\left(\mathrm{CI}_{90}=0.058-0.078 ; \chi^{2}=246.65, d f=85, p<0.0001\right)$. Results from this analysis are depicted 
in Figure 4. Pathways that were not significant are depicted with a grey, dotted line. Unexpectedly, the direct effect from Acceptance to Non-attachment was negative (standardized estimate $=-0.44, z=-4.12$ ), indicating that respondents who reported greater acceptance about their internal lives also reported an increased sense that their happiness was a function of external circumstances in their lives. Non-attachment and Acceptance were related to all other variables in the expected directions. Another noteworthy finding in the model was the positive association between Attention and Psychological distress (standardized estimate $=0.14, z=2.45$ ), indicating that an increased tendency to pay attention to one's present-moment experience was associated with increased self-reported distress.

A second model tested only the original, hypothesized paths and any significant paths from the all-inclusive model. This model included one hypothesized path coefficient that was found to be non-significant in the preceding analyses (i.e., the direct effect from Negative emotion regulation to Rumination) because eliminating other non-significant parameters can allow a non-significant path coefficient to become significant. This model also included all significant coefficients from the all-inclusive model. The model excluded path coefficients that were not hypothesized and not found to be significant in the previous, all-inclusive model (i.e., the following direct effects: Attention to Clarity, Negative emotion regulation, and Rumination; Acceptance to Psychological distress; Clarity to Non-attachment; and Nonattachment to Psychological distress).

This model produced an RMSEA of $0.067\left(\mathrm{CI}_{90}=0.057-0.076 ; \chi^{2}=255.74, d f=91\right.$, $p<0.0001)$. Results from this analysis are depicted in Figure 5. The hypothesized direct effect from Negative emotion regulation to Rumination was not significant $(z=-1.15, p=0.25)$, but 
all other hypothesized path coefficients were significant, as were all other significant parameters from the all-inclusive model. The correlation between Attention and Acceptance was not significant $(r=-0.10, z=-1.53, p=0.13)$.

A summary of the standardized indirect and total effects are presented in Tables 9 and 10 , respectively. Indirect effects represent the effect that one variable exerted on another variable via intervening variables. (Direct effects are simply the parameter estimates in a model for the coefficient of the path connecting two variables; these are depicted in Figure 5.) Total effects summarize the cumulative effect that one variable exerted on another variable via both direct and indirect effects. Table 10 reveals that every one-standard-unit change in Acceptance was associated with a 0.67-standardized-unit total increase in the ability to regulate negative affect and a 0.55 -standardized-unit total decrease in Psychological Distress. In contrast, Attention had a much smaller effect on the other variables in the model. Every one-standard-unit shift in Attention was associated with a 0.03 -standard-unit total increase in Negative Emotion Regulation and a 0.07-standard-unit total increase in Psychological Distress. Furthermore, the tables illustrate the complex relationship between Attention and Psychological Distress in participants without mindfulness training. Attention exerted a (non-significant) positive total effect on Psychological Distress, but a negative indirect effect on Psychological Distress. In other words, an increased tendency to attend to present-moment experience was associated with greater Psychological Distress, overall, but nonetheless this tendency impacted other variables in the model in such a way that they simultaneously diminished Psychological Distress. These tables also highlight the prominent role that acceptance of one's internal experience and the ability to regulate negative affect 
play in psychological distress. Both variables individually had a larger effect on

Psychological Distress than did all other variables combined.

Step 3: Mechanisms of Action in the Relationship between Mindfulness and Flourishing Mental Health

To explore the possibility that mindfulness might influence well-being via the same mechanisms by which it influences measures of psychological distress, two models were tested in which flourishing mental health was the final outcome variable in the model. A third model explored mechanisms of action when both Psychological Distress and Flourishing were in the model together. In these models, the Short Flourishing measure and the Satisfaction with Life measure were allowed to load together on a Flourishing factor.

The first model examined all possible path coefficients when Flourishing mental health was the final outcome variable. This model produced an acceptable fit for the data (Figure 6; RMSEA $=0.074 ; \mathrm{CI}_{90}=0.065-0.084 ; \chi^{2}=278.29, d f=85, p<0.0001$ ). The pattern of significant paths was almost identical in this model to those when Psychological Distress was the final outcome variable. The one exception was the direct effect from Non-attachment to Flourishing $(z=3.95, p=0.0001)$, which was significant in this model, indicating that Non-attachment was associated with increased Flourishing, independently of its impact on Rumination, which also influenced Flourishing.

A second model eliminated the non-significant paths from the previous model (i.e., the direct effects from Attention to Negative Emotion Regulation, Non-attachment, and Rumination, from Acceptance to Flourishing, from Clarity to Non-attachment and Rumination, and from Negative Emotion Regulation to Rumination). This model produced a negligible improvement in model fit (Figure 7; RMSEA $=0.073 ; \mathrm{CI}_{90}=0.064-0.082$; 
$\left.\chi^{2}=294.09, d f=92, p<0.0001\right)$. Interestingly, in both of these models, Attention exerted a positive impact on Flourishing, which is consistent with hypotheses about how mindfulness works but inconsistent with findings from the models explored in Step 2, above, in which increased Attention was associated with increased Psychological Distress.

To better understand the complex relationship between Attention, Flourishing, and Psychological Distress, a third and final structural equation model was tested in which Flourishing and Psychological Distress were both included as separate outcome variables. ${ }^{10}$ This model fit the data slightly better than did the two models with Flourishing alone $\left(\mathrm{RMSEA}=0.071 ; \mathrm{CI}_{90}=0.063-0.080 ; \chi^{2}=360.68, d f=116, p<0.0001\right)$. This model is presented in Figure 9. In the figure, Psychological Distress and paths that directly influence Psychological Distress are colored burgundy, whereas Flourishing and the paths that directly influence it are colored teal. Significant path coefficients are denoted with a solid line, whereas non-significant path coefficients are depicted with a dotted line. Paths that are significant but do not differ from previous models and are not germane to understanding how the model fits when both Flourishing and Psychological Distress are included together are featured in grey. For visual clarity, path coefficients are only included for the direct effects from Attention to Psychological Distress and from Attention to Flourishing.

As can be seen in Figure 8, the variables that significantly predict Psychological Distress and Flourishing are identical regardless of whether Psychological Distress and Flourishing are considered in isolation or together in one model. In particular, Attention 
exerted significant direct effects on both Psychological Distress and Flourishing, whereas Acceptance did not significantly predict either variable. Clarity, Negative Emotion Regulation, and Rumination exerted significant direct effects on both outcome variables. Lastly, Non-attachment significantly predicted Flourishing but did not significantly predict Psychological Distress. As with previous models, Attention and Acceptance were not significantly correlated $(r=-0.10, z=-1.51, p=0.13)$

The total effects for this model are featured in Table 11. Acceptance exerted a significant, negative total effect on Psychological Distress $(z=-8.70, p<0.0001)$ and a significant, positive total effect on Flourishing $(z=5.67, p<0.0001)$, despite its nonsignificant direct effects on these variables. In contrast, Attention exerted significant, positive direct effects on both Psychological Distress $(z=2.44, p<0.02)$ and Flourishing $(z=2.16, p$ $=0.03)$. The total effect Attention exerted on Psychological Distress was not significant $(z=$ $1.15, p=0.25)$, however, reflecting the influence of the negative, indirect effect Attention had on Psychological Distress via other variables in the model. Attention did exert a significant total effect on Flourishing $(z=3.25, p=0.001)$.

As expected, Psychological Distress and Flourishing were negatively correlated $(r=-$ 0.52). The model explained 60\% of the variance in Psychological Distress and 55\% of the variance in Flourishing.

\footnotetext{
${ }^{10}$ Previous research indicates that positive and negative psychological adjustment are distinct constructs. To confirm that this was the case in this sample, a confirmatory factor analysis was conducted in which the two indicators for Psychological Distress and the two indicators for Flourishing were allowed to load on a general "psychological adjustment" factor. This model produced a poor fit for the data (RMSEA $=0.32 ; \mathrm{CI}_{90}=0.26-$ $0.38 ; \chi^{2}=83.61, d f=2, p<0.0001$ ). In contrast, a two-factor model, consisting of a "Psychological distress" factor and a "Flourishing" factor, fit well (RMSEA $=0.00 ; \mathrm{CI}_{90}=0.00-0.12 ; \chi^{2}=0.64, d f=1, p=0.42$ ).
} 


\section{Brief Discussion of Study 2}

Study 2 replicated in an independent sample the common factors in mindfulness and emotion regulation measures identified in Study 1. Study 2 then expanded upon these findings to test an enriched model for mindfulness' potential mechanisms of action that modeled mindfulness and its most proximal sequelae (i.e., clarity and negative emotion regulation) more accurately. In addition, Study 2 integrated these findings with other possible mechanisms of action, specifically non-attachment and rumination, by which mindfulness might influence outcome variables such as psychological distress and positive psychological adjustment.

Results from this model confirmed the hypothesis that negative emotion regulation, non-attachment, and rumination remained important mechanisms of action when mindfulness was modeled as two distinct components encompassing present-centered attention and acceptance of one's experience, which both facilitated clarity about one's internal life. Results also revealed that Attention and Acceptance were clearly distinct constructs in a sample with no formal mindfulness training. Of particular importance, Acceptance exerted much stronger effects on other variables in the model than did Attention, indicating its relatively greater importance to Psychological Distress and Flourishing and related constructs. Attention exhibited a complex relationship to Psychological distress, in which it simultaneously directly augmented distress and indirectly diminished it. Attention did not exhibit a similarly complex and paradoxical relationship to Flourishing: Attention was both directly and indirectly associated with increased Flourishing. Lastly, results confirmed the hypothesis that mindfulness influences both positive and negative psychological adjustment via the same mechanisms, despite the fact that these are distinct aspects of mental health. 


\section{GENERAL DISCUSSION}

The two studies presented here contribute to an enriched understanding of mindfulness, as a construct, and the mechanisms by which naturally-occurring individual differences in mindfulness influence both positive and negative measures of mental health. The first study contributes to a more precise understanding of the component processes that comprise current conceptualizations of mindfulness and emotion regulation, and how these processes are related to each other. Results suggested that current measures for mindfulness and emotion regulation assess a heterogeneous and overlapping set of constructs. Specifically, measures for mindfulness and emotion regulation were not well-modeled by separate "mindfulness" and "emotion regulation" factors, indicating that they are not homogenous constructs. Instead, exploratory factor analyses suggested that questionnaires for mindfulness and emotion regulation jointly measure three underlying constructs. These three common constructs can be described as an accepting and non-judgmental orientation towards one's internal experience, clarity about one's internal experience, and the ability to regulate negative affect. The first construct, involving acceptance of internal experience, is included in most definitions of mindfulness (e.g., Bishop et al., 2006). The last construct, involving the ability to regulate negative affect, is closest to conventional definitions of emotion regulation (Gross, 1998).

These results suggest, first, that multiple sub-processes may be subsumed under the terms "mindfulness" and "emotion regulation." Second, in an attempt to design comprehensive measures for mindfulness and emotion regulation, distinct, albeit related, 
processes may have been absorbed into both of these terms. Aspects of these terms appear to have become commingled over time. This work suggests that it may not be appropriate to use the FFMQ and the DERS, as they are designed, to measure "mindfulness" and "emotion regulation." Instead, using a combination of subscales from both questionnaires might most accurately reflect the constructs that underlie these terms.

These findings call into question which of the FFMQ subscales or common factors should be considered "mindfulness." For guidance, the present studies used the operational definition for mindfulness proposed by Bishop et al. (2004), which posits that mindfulness is composed of an attentional component and an attitudinal, acceptance-based component. These two components are common to almost every existing definition for mindfulness. It should be noted that the models presented here are an acceptable fit for the data, regardless of whether the superordinate term "mindfulness" is applied to some of the constructs in the models (i.e., Attention and Acceptance). Nonetheless, in terms of understanding mindfulness and its proximal sequelae, these models apply to a specific operational definition for mindfulness and might not fit as well if mindfulness were defined differently.

Results from the first study also suggested that some of the constructs assessed with current mindfulness and emotion regulation measures may be sequelae of others. In particular, a greater tendency to notice internal and external aspects of one's present-moment experience and an increased willingness to acknowledge and refrain from judging that experience, even when it was painful, were associated with an increased ability to identify and label emotional experience. This ability was in turn associated with an improved ability to manage negative affect. Present-centered attention and acceptance also directly influenced the ability to regulate negative affect. Thus, in addition to helping disentangle the different 
constructs measured by mindfulness and emotion regulation questionnaires, this work also helps model how these constructs might be related to each other.

Lastly, the first study replicated previous research on the lack of association between present-centered attention and a non-judgmental approach towards one's experience in samples with no formal mindfulness training (Baer et al., 2006). This highlights the importance of treating these two dimensions of mindfulness as separate and distinct constructs, which may not be related when they are considered as naturally-varying individual differences. Despite this, higher levels of both were associated with an increased ability to recognize what one was feeling and effectively manage this affect when it was negative. Acceptance exerted stronger effects on both clarity about one's internal experience and the ability to regulate negative affect than did present-centered attention, indicating that it is more important to these abilities than is attention.

Study 2 used the findings from Study 1 on the nature of mindfulness to more accurately model the mechanisms by which naturally-varying, dispositional mindfulness might influence two aspects of mental health, psychological distress and flourishing mental health. This study produced several findings. First, results provided support for the hypothesis that dispositional, naturally-varying mindfulness impacts mental health by first impacting other constructs, which in turn impact mental health. In particular, this study helped identify some of the mediating constructs between mindfulness and mental health. These include clarity about one's internal experience, the ability to regulate negative affect, the ability to view one's happiness as independent of external circumstances, and rumination. Acceptance of one's experience influenced psychological distress and flourishing to the extent that it impacted the mediators, which in turn influenced psychological distress and 
flourishing. The relationship between present-centered attention and mental health was more complex and is discussed in greater detail below, but present-centered attention exerted a portion of its impact on mental health via the impact it had on clarity about one's internal experience, the ability to regulate negative affect, the ability to view one's happiness as independent of external circumstances, and rumination.

Second, despite the fact that psychological distress and flourishing mental health are distinct aspects of mental health, mindfulness and the mediators explored here appear to influence these distinct aspects of mental health via virtually identical mechanisms. This suggests that although remediation of psychological distress is not the same thing as creating flourishing mental health, the same processes act equally on both. The abilities, such as recognizing what one is feeling, managing negative affect, etc., that must be addressed to treat depression and anxiety may be identical to those necessary to help a non-distressed person achieve even greater life satisfaction. Flourishing mental health and psychological distress were correlated at $r=-0.52$. The moderately strong correlation is consistent with their shared dependence on some of the same processes, and also with their distinctiveness as psychological outcomes.

Third, one of the most noteworthy and intriguing findings in the study was the paradoxical relationship between present-centered attention and psychological distress. Directly attending to one's present moment experience both decreased psychological distress, by beneficially impacting other constructs which then decreased distress, and increased psychological distress, via a direct association. Taken cumulatively, these two simultaneous influences cancelled each other out, such that a participant's tendency to notice his or her experience did not affect his or her levels of psychological distress. 
One explanation for the paradoxical relationship between present-centered attention and psychological distress is that directly attending to one's experience may make that experience more salient in awareness. Individuals who attend to their experience may be more aware of the times they feel anxious and depressed, as well as the times they feel satisfied with their lives and fulfilled. This awareness may intensify negative affect in the short-term, but it could also provide individuals with information they can use to better manage their internal lives. For example, a tendency to notice internal and external stimuli might make a person more aware of her depression or anxiety, but it might simultaneously provide information about the context in which the emotion has arisen, the effectiveness of emotion regulation strategies, reactions to other, potentially positive aspects of one's experience, and other information that could down-regulate present and future distress. Thus, at the same time that directly attending to negative affect may augment it in the moment, it may simultaneously provide information that ultimately serves to diminish it.

A fourth noteworthy finding from both studies was the greater importance to mental health of accepting one's experience, and refraining from judging it, relative to simply attending to it. Acceptance of one's experience was more important to both positive and negative psychological functioning than was any other constructs in the model except the ability to regulate negative emotions. (The ability to regulate negative emotions was itself more strongly influenced by acceptance than by any of the other preceding variables in the model, in both studies.)

Increases in acceptance were associated with improved functioning on all constructs except non-attachment. Acceptance was unexpectedly directly associated with a greater sense that one's happiness is a function of external circumstances. At the same time, acceptance 
was also associated with increased clarity about one's internal experience and an increased ability to regulate negative affect, which in turn predicted an increased sense that one's happiness is internally determined and independent of external circumstances. Thus, as with the relationship between present-centered attention and psychological distress, acceptance was directly associated with less non-attachment at the same time that it was indirectly associated with greater non-attachment. These two influences balanced each other such that acceptance did not exert a significant total effect on non-attachment.

Examination of the questionnaire items for the Linking Inventory, which was used to measure non-attachment, provides some insight into a possible explanation for this relationship. The Linking Inventory examines the extent to which respondents "link" their happiness to specific external circumstances in their lives, with response choices that indicate a more independent relationship between external circumstances and happiness reflecting non-attachment. Some of the questionnaire items are sufficiently extreme, however, that it would be unlikely for a respondent to endorse the more non-attached position in the absence of training or a specific practice intended to cultivate this orientation. For example, one item asks "Think about the things in your life that you really want, but just can't get. Maybe you want to be a doctor, but you realize that your grades are not going to be good enough to get into medical school. Or maybe you want to go out with a certain person, but that person won't go out with you. How does this affect your happiness?" The response choices are "The more things I want but can't get, the less happy I am," (reflecting less non-attachment) and "Wanting things I can't get does not make me less happy" (reflecting more non-attachment). Respondents who are not intentionally cultivating equanimity may be very unlikely to indicate that disappointed life aspirations or unrequited affection would not adversely impact 
their happiness, if they are honest with themselves. The negative association between acceptance of internal experience and non-attachment may reflect an increased propensity among those who are more accepting of themselves to be honest with themselves, even about painful realities. Endorsement of the "healthier," more non-attached response option on questions like this on the Linking Inventory may reflect defensiveness (Boden, Hyland, \& Dale, 2005) or social desirability (Crowne \& Marlow, 1960), rather than genuine nonattachment. Thus, more accepting respondents might report less non-attachment at the same time that they report greater clarity about their internal experience, an increased ability to manage negative internal experience, and better overall psychological adjustment.

Another possibility is that the negative association between acceptance and nonattachment reflects the influence of a third, unmeasured variable. Specifically, participants who have rigid standards for how the world "should" be may be both more critical of themselves when they do not feel the way they think they should (reflecting low acceptance) and may also be more likely to believe that happiness "should" be independent of life circumstances (which would appear to be a more non-attached position).

\section{$\underline{\text { Implications for Clinical Work }}$}

Results from these studies have implications for clinical work. First, in samples with no formal mindfulness training, an accepting and nonjudgmental orientation towards one's internal experience is clearly more beneficial for mental health than is simply paying attention to one's experience. Not only did acceptance exert larger total effects on almost all variables in the model than did present-centered attention, but its effects were almost exclusively beneficial. (The one exception to this was the negative association between 
acceptance and non-attachment.) This suggests that distressed clients may benefit more from acceptance-based practices, such as identifying and reframing judgmental thoughts, than from purely attentional practices, such as doing one thing at a time or noticing internal or external stimuli.

Second, it should be noted that present-centered attention did positively impact flourishing mental health, and did indirectly benefit psychological distress. Thus, attentional practices may still be beneficial for distressed clients. In recognition of the complex relationship between present-centered attention and mental health, it may be important to pair attentional practices with acceptance-based practices and monitor the impact attentional practices have on psychological distress.

Third, these results also suggest possible foci for intervention when clients are distressed or experiencing sub-optimal flourishing mental health. One can work backwards in the model to identify factors that may contribute to the client's present difficulties. For example, distressed clients are more likely to be ruminating and having difficulty managing negative affect. If so, helping the client identify what $\mathrm{s} / \mathrm{he}$ is feeling may be beneficial. These results indicate that two strategies that may be of assistance in this endeavor would be to help the client attend to his or her experience, and to help the client refrain from judging his or her internal experience.

Fourth, the composition of the clarity and negative emotion regulation factors also has implications for clinical work. In particular, the ability to label experience with words loaded on the same factor as did measures associated with the ability to identify what one was feeling. This suggests that being able to use language to describe one's experience is closely related to knowledge of one's experience. A client who claims that s/he knows what 
$\mathrm{s} /$ he is feeling but cannot describe it may not actually understand the emotion. Furthermore, this client is also likely to struggle with how to manage the emotion.

Similarly, the ability to regulate negative affect included subscales related to being able to engage in goal-directed behavior, control behavior, generate strategies to feel better, think positive thoughts, and observe distress without reacting to it. The presence of this last indicator suggests that the ability to effectively manage negative affect requires a certain amount of distance from and equanimity in the face of psychological distress. This distance appears to be part of the ability to manage the affect by thinking positive thoughts, utilizing coping strategies, engaging in constructive behavior, and inhibiting unconstructive impulses. Thus, clients who are fused with their distress and unable to identify it as distinct from a larger part of themselves are unlikely to be able to regulate the emotion. Working backwards in the model, helping the client identify and label the emotion is likely to help.

\section{$\underline{\text { Limitations and Future Directions }}$}

There are two primary limitations associated with this work, which suggest promising avenues for future inquiry. First, these studies examined mindfulness solely as a dispositional, naturally-varying difference. Previous work has found dispositional differences in mindfulness to be associated with meaningful outcomes, such as global measures of mental health. Therefore, the effort to identify how dispositional mindfulness might influence mental health is an important contribution to understanding of both mindfulness and mental health. The possibility also remains that mindfulness may work by different mechanisms when it is manipulated via a mindfulness-based intervention or meditation practice. Although the findings presented here are consistent with theoretical work describing how an active 
mindfulness intervention might influence different aspects of mental health, the model should be tested in different samples, such as participants undergoing a meditation class or psychotherapy clients enrolled in a mindfulness-based intervention. This is necessary to confirm that both the factor structure and structural relationships presented here generalize to participants who are attempting to increase their mindfulness. In particular, the relationship between present-centered attention and psychological distress may be different in those with mindfulness training. In addition, experimental designs are needed to provide more definitive tests of the proposed causal mechanisms in this model.

A second limitation associated with this work is the use of self-report measures. With self-report measures, participants may report their theories about themselves, rather than their actual functioning. The model presented here is consistent with past empirical and theoretical work on mindfulness' mechanisms of action. Nonetheless, it should be tested with behavioral and other-report measures to ensure that it is not modeling participants' imagined functioning, rather than their actual functioning. Other-report measures may be particularly relevant to assessing aspects of negative emotion regulation, including the extent to which a participant can engage in goal-directed behavior, refrain from feeling helpless and passive, and refrain from impulsive and maladaptive behavior in the presence of negative affect. Behavioral measures may be particularly useful in assessing present-centered attention (discussed below).

Two of the self-report measures employed in this work, the FFMQ Observe subscale and the Linking Inventory, may be subject to particular difficulties. The FFMQ Observe subscale assesses the extent to which respondents regularly attend to their present-moment experience, including physical sensations and environmental stimuli. An inherent confound 
exists in asking participants who may not regularly notice their present-moment experience to report how frequently they do this: they may not be paying attention enough to know that they are not paying attention. There is some work indicating that self-reported mindfulness scores initially drop when participants undertake a mindfulness practice (Pradhan et al., 2007). This initial drop is generally considered to reflect the participants' growing awareness of how mindless they often are. Similarly, when mindfulness is considered as a naturallyvarying individual difference, extremely mindless participants may not be aware of how distracted they are and may report more mindfulness than participants who are, in actuality, more mindful. For this reason, behavioral measures for present-centered attention may be a better way to assess this construct. One possible measure involves asking participants to read text and report at regularly intervals whether or not they are attending to the task, which would test the respondent's mindfulness to the task versus mind-wandering (Smallwood \& Schooler, 2006). A second possibility asks participants to press a computer key with each exhalation, while physiological equipment tracks participants' breaths and identifies whether each breath is matched by a press on the computer key (Ekblad et al., in preparation). Unnoted breaths reflect mindlessness.

The Linking Inventory, which was used to measure non-attachment in this work, may also be subject to additional difficulties, in addition to those inherent in self-report measures. The dichotomous, forced-choice response options on the Linking Inventory may pull for extreme responding and distort participants' actual positions. This may have contributed to the unexpected, negative direct effect between acceptance of internal experience and nonattachment. Furthermore, some of the questions themselves may be extreme and unlikely to capture genuine, naturally-varying differences in non-attachment in a sample with no formal 
practice related to the construct. To the author's knowledge, there are currently no other measures with which to measure non-attachment, despite the fact that researchers in this area have suggested that non-attachment to outcome is central to understanding how mindfulness works (Kabat-Zinn, 2003). Construction of additional measures for non-attachment would be a substantial contribution to the field. The results presented here should be replicated using other measures for non-attachment to ensure that the current results are not an artifact of the specific measure used in this study.

The present model is not intended to be a complete and exhaustive representation of potential mediators in the relationship between mindfulness and mental health. In addition to addressing the limitations associated with the present work, future studies might investigate other possible mechanisms of action in this relationship. One such mechanism is exposure and desensitization to unpleasant emotional experiences (Baer, 2003; Brown, Ryan, \& Creswell, 2007; Shapiro et al., 2006). This proposed mechanism assumes that distressing thoughts and emotions are a form of feared stimulus for many people (Linehan, 1993). These thoughts are then avoided, which negatively reinforces the fear behavior and perpetuates phobic reactions to these stimuli. Nonjudgmentally attending to distressing thoughts or emotions, in the absence of negative consequences, may result in desensitization to these kinds of thoughts and emotions. Furthermore, an increased ability to tolerate these stimuli may result in less emotional reactivity to them. This hypothesis is consistent with theoretical and empirical work on how exposure therapy works with other kinds of anxiety-provoking stimuli (McLean \& Woody, 2001). This hypothesis is also consistent with the significant positive correlation, in the work presented here, between the FFMQ subscales associated with nonreactivity to internal experience and observation of experience, as well as the 
significant positive correlations between the FFMQ Nonreactivity subscale and each of the subscales associated with acceptance of internal experience.

Another possible mechanism is decentering ${ }^{11}$ (Baer, 2003; Brown, Ryan, \& Creswell, 2007; Shapiro et al., 2006; Teasdale, 1999). Decentering involves the recognition that one's thoughts may not necessarily reflect reality. This awareness is hypothesized to allow a person to disengage from habitual thought patterns and perceive the world in different, and potentially more adaptive, ways. When habitual thought patterns are negative, this skill can assist in attenuating the connection between negative thoughts and negative affect. For example, although a person may still have the thought, "I'm depressed and I'll always be miserable," the person may also be able to identify that this thought issues from their current affect may only reflect their current mood, rather than objective reality. MBCT is hypothesized to work in part via this mechanism (Teasdale et al., 1995).

\section{$\underline{\text { Summary }}$}

The two studies presented here contribute to greater conceptual clarity about what mindfulness and emotion regulation are, and how these constructs are related to each other. This work indicates that some constructs that are subsumed under the term mindfulness may be more accurately thought of as sequelae of mindfulness. Furthermore, this work proposes and tests several mechanisms of action that might explain how dispositional, naturallyvarying mindfulness influences both positive and negative psychological adjustment. The ability to identify and differentiate among emotions, successfully regulate negative emotions, refrain from linking one's happiness to external circumstances, and refrain from ruminating

\footnotetext{
${ }^{11}$ Also called "reperceiving" or "deautomatization."
} 
appear to be important paths by which present-centered attention and acceptance of experience, together considered mindfulness in this work, impact mental health. Results indicate that mindfulness influences positive psychological adjustment via the same mechanisms by which it influences negative psychological adjustment. Lastly, this work points to a complicated and counter-intuitive relationship between a critical aspect of mindfulness, present-centered attention, and psychological distress in samples with little formal mindfulness training. These results suggest that a greater tendency to observe one's experience is not necessarily beneficial for psychological distress for those without formal mindfulness training. Thus, these results provide important insight into the relationship between mindfulness and mental health in the absence of an intervention intended to alter any of the constructs examined here. 


\section{APPENDICES}

Appendix A: Additional Models Tested in Study 1

One limitation with structural equation models is the possibility that multiple models may fit the data equally well. In addition, the failure of the FFMQ Acting with Awareness subscale to load on any common factor admitted the possibility that it might fit into the proposed model (Figure 3) in a variety of ways. A series of non-theory-driven models explored this possibility. It should be noted that the practice of generating and testing nontheoretical models, in an attempt to produce a well-fitting model, is controversial. This practice is generally considered to be post-hoc and less compelling evidence for the pattern of relationships among variables that are theory-driven and hypothesized models. Nonetheless, given the fact that mindfulness has often been measured with a questionnaire (i.e., the MAAS) that is very similar to the FFMQ Acting with Awareness subscale, this was an important set of exploratory analyses.

A sequence of models, depicted in Figures 9 - 12, introduced Acting with Awareness at different junctures in the existing model. Given the absence of a theoretical reason to do otherwise, and to optimize the chances of finding a good model fit, all structural pathways between the factors were freed in the models. None of these models fit as well as did the model featured in Figure 5. A last model explored the possibility that Acting with Awareness is a third and distinct aspect of mindfulness, in addition to Attention and Acceptance. This model also did not fit as well as the model featured in Figure 5.

Two aspects of the results from these models warrant commentary. First, in all three of the models in which Attention exerted a direct effect on Acting with Awareness, this path 
coefficient was not significant. The absence of a significant relationship between these two FFMQ subscales (i.e., the FFMQ Observe subscale, which defines the Attention factor, and the FFMQ Acting with Awareness subscale) is curious because deliberately attending to one's behavior might appear to be an aspect of deliberate attending to one's present-moment experience, in general. The failure of these two subscales to load on the same factor, and the absence of a relationship between these constructs in the models we investigated here, suggests that this is not the case, at least in samples with no mindfulness training.

An additional noteworthy finding is the absence of a significant relationship, in all models tested, between Acting with Awareness and Negative Emotion Regulation. This is particularly interesting because both constructs tend to be behaviorally-oriented: the Acting with Awareness subscale consists of items that involve attentiveness to one's actions, whereas the Negative Emotion Regulation factor is heavily weighted towards measures that emphasize behavioral control. The fact that Acting with Awareness did not significantly predict Negative Emotion Regulation in any model suggests that, surprisingly, attentiveness to one's behavior is not associated with increased behavioral control in this sample.

This non-significant relationship is also surprising because the recent interest in mindfulness-based clinical interventions is driven, in part, by the supposition that mindfulness facilitates management of negative affect. This is consistent with a rich history of theoretical work on the benefits of mindfulness in Eastern philosophy. Furthermore, all three of the other constructs that include subscales from the mindfulness FFMQ measure (i.e., Attention, Acceptance, and Clarity) are significantly associated with Negative Emotion Regulation. Although it is possible that the non-significant relationship between Acting with Awareness and Negative Emotion Regulation is a function of the sample, which consisted 
largely of participants with no mindfulness training, one might expect that if Acting with Awareness does facilitate regulation of negative affect, this relationship would nonetheless be present when these constructs are examined as individual differences. It is also possible that intentional, conscious behavior reflects global mindfulness and is in some way a summary for multiple constructs represented in the model (Figure 3), rather than an additional element. Nonetheless, were this true, one might still expect a significant relationship between Acting with Awareness and Negative Emotion Regulation. Thus, this curious finding raises more questions than it answers about the relationship between these constructs. Given the unclear relationship between Acting with Awareness and the other variables in the model in Study 1, and the absence of a theory-driven reason to do otherwise, this variable was not included in the models tested in Study 2. 


\section{Appendix B: Additional Analyses with Attention and Acceptance}

The paradoxical and un-hypothesized relationship between Attention and Psychological Distress raised several questions, particularly given the hypothesized relationship between Attention and other variables in the model, including Positive Psychological Adjustment. One possible explanation for these findings was the presence of an interaction between Acceptance and Attention, whereby Attention might influence Psychological Distress differently at different levels of Acceptance. Four multiple regression models were tested to examine this possibility. In each model, Attention (measured by the FFMQ Observe subscale), one of the Acceptance indicators (either the DERS Acceptance subscale or the FFMQ Nonjudging subscale), and their interaction predicted either the BSI Depression subscale or BSI Anxiety subscale, for a total of four models. ${ }^{12}$

The interaction of attention and acceptance was significant for only one of these four models. Specifically, when Acceptance was measured with the DERS Acceptance subscale, the interaction of this variable with the FFMQ Observe subscale significantly predicted BSI Anxiety $(t=-2.63, d f=1, p<0.01)$. This significant relationship did not hold when Acceptance was measured using the FFMQ Nonjudging subscale, nor when BSI Depression was the dependent variable. Probing the significant interaction of the FFMQ Observe subscale and the DERS Acceptance subscale revealed that the FFMQ Observe subscale significantly positively predicted BSI Anxiety at one standard deviation above the mean for DERS Acceptance $(t=3.26, d f=1, p<0.01)$ and at the mean for DERS Acceptance $(t=$ 2.35, $d f=1, p<0.02)$, but not at one standard deviation below the mean for Acceptance ( $t=$ - 
$0.22, d f=1, p=0.82)$. In other words, at average or high levels of acceptance, presentcentered attention predicted greater self-reported anxiety. At low levels of acceptance, there was no relationship between present-centered attention and self-reported anxiety.

Examination of the individual measures for these variables provides insight into a possible explanation for this relationship. The FFMQ Observe subscale, which was used here to measure present-centered attention, includes several items that focus on awareness of bodily sensations, such as those experienced when walking, bathing, or eating. The BSI Anxiety subscale includes questions that emphasize the physiological correlates of anxiety, such as feeling restless, tense, or a feeling of "shakiness" inside. The DERS Awareness subscale assesses reactions to negative emotions that are extremely rejecting, such as feeling guilty, ashamed, embarrassed, angry, or weak because one is upset. It is possible that individuals who regularly attend to their physical sensations, as measured by the FFMQ Observe subscale, are also more likely to notice internal indicators of agitation. It may also be that only individuals who are receptive to this information, and not judgmental of their experience as reflected by the DERS Awareness subscale, identify their anxiety.

Interestingly, acceptance of experience did not moderate the impact of presentcentered awareness on anxiety when it was measured with the FFMQ Nonjudging subscale. The FFMQ Nonjudging subscale includes items related to telling oneself that thoughts are good or bad, or criticizing one's reactions, but these items are not phrased as strongly as are the DERS Acceptance statements about feeling ashamed, angry, or embarrassed about one's

\footnotetext{
${ }^{12}$ An additional four models examined the possibility of an interaction between present-centered attention and one of the acceptance measures when either Satisfaction with Life or Flourishing was the dependent variable, even though the relationships between Attention, Acceptance, and Positive Psychological Adjustment were all as hypothesized in the structural equation models. No significant interactions were found.
} 
emotional experience. Thus, only a very punitive or rejecting orientation towards negative internal experiences may interfere with accurate identification of physiological information. 
TABLES 
Table 1. Descriptive Statistics and Indicator Inter-correlations (Study 1)

\begin{tabular}{|c|c|c|c|c|c|c|c|c|c|c|c|c|c|c|c|}
\hline & & Std. & & & & & & & & & & & & & \\
\hline Indicator & Mean & Dev. & 1 & 2 & 3 & 4 & 5 & 6 & 7 & 8 & 9 & 10 & 11 & 12 & 13 \\
\hline 1. FFMQ Observe & 3.22 & 0.57 & 1 & & & & & & & & & & & & \\
\hline 2. DERS Awareness & 3.64 & 0.67 & .34 & 1 & & & & & & & & & & & \\
\hline 3. FFMQ Nonjudging & 3.19 & 0.70 & -.15 & .11 & 1 & & & & & & & & & & \\
\hline 4. DERS Acceptance (squared) & 3.80 & 0.88 & .00 & .27 & .55 & 1 & & & & & & & & & \\
\hline 5. FFMQ Describe & 3.29 & 0.65 & .10 & .37 & .20 & .28 & 1 & & & & & & & & \\
\hline 6. TMMS Clarity & 3.43 & 0.59 & .08 & .42 & .41 & .49 & .64 & 1 & & & & & & & \\
\hline 7. DERS Clarity (squared) & 3.80 & 0.66 & .11 & .44 & .39 & .48 & .58 & .82 & 1 & & & & & & \\
\hline 8. FFMQ Nonreactivity & 2.98 & 0.54 & .17 & .02 & .14 & .25 & .15 & .29 & .25 & 1 & & & & & \\
\hline 9. TMMS Repair (squared) & 3.56 & 0.72 & .11 & .25 & .21 & .29 & .15 & .36 & .32 & .33 & 1 & & & & \\
\hline 10. DERS Goals & 2.85 & 0.91 & .01 & -.01 & .25 & .31 & .23 & .32 & .26 & .33 & .24 & 1 & & & \\
\hline 11. DERS Impulse Control (squared) & 4.13 & 0.77 & .11 & .22 & .32 & .47 & .20 & .44 & .42 & .40 & .47 & .39 & 1 & & \\
\hline 12. DERS Strategies (squared) & 3.90 & 0.76 & .09 & .18 & .39 & .56 & .24 & .53 & .50 & .45 & .58 & .54 & .71 & 1 & \\
\hline 13. FFMQ Acting with Awareness & 3.16 & 0.61 & -.01 & .14 & .29 & .15 & .15 & .27 & .27 & .06 & .09 & .29 & .25 & .25 & 1 \\
\hline
\end{tabular}


Notes. Correlations $=0.11$ or larger are significant at $\mathrm{p}<0.05$. Means and standard deviations are reported in their original, rather than their transformed, units. 
Table 2. Exploratory Factor Analysis with Three Factors and All Potential Indicators (Study

1)

\begin{tabular}{lccc}
\hline & \multicolumn{3}{c}{ Factor } \\
Indicator & 1 & 2 & 3 \\
\hline FFMQ Observe & .113 & .126 & .316 \\
DERS Awareness & -.038 & .540 & .089 \\
FFMQ Nonjudging & .150 & .186 & -.758 \\
DERS Acceptance (squared) & .386 & .214 & -.386 \\
FFMQ Describe & -.112 & .764 & .019 \\
TMMS Clarity & .133 & $\mathbf{. 8 2 8}$ & -.104 \\
DERS Clarity (squared) & .114 & $\mathbf{. 8 0 1}$ & -.102 \\
FFMQ Nonreactivity & $\mathbf{. 5 1 1}$ & -.009 & .087 \\
TMMS Repair (squared) &. $\mathbf{6 1 1}$ & .011 & .066 \\
DERS Goals & .550 & -.026 & -.064 \\
DERS Impulse Control (squared) &. $\mathbf{7 3 6}$ & .010 & -.033 \\
DERS Strategies (squared) & $\mathbf{. 9 6 8}$ & -.048 & -.042 \\
FFMQ Acting with awareness & .136 & .170 & -.197 \\
\hline
\end{tabular}


Table 3. Exploratory Factor Analysis with Four Factors and All Potential Indicators (Study

1)

\begin{tabular}{lcrrr}
\hline & \multicolumn{5}{c}{ Factor } \\
Indicator & 1 & 2 & 3 & 4 \\
\hline FFMQ Observe & .368 & .113 & -.019 & -.198 \\
DERS Awareness & .941 & -.163 & -.170 & .215 \\
FFMQ Nonjudging & -.077 & .035 & -.046 &. $\mathbf{8 2 5}$ \\
DERS Acceptance (squared) & .092 & .298 & -.091 & .492 \\
FFMQ Describe & .033 & -.091 & -.0754 & -.058 \\
TMMS Clarity & -.022 & .120 & -.861 & .062 \\
DERS Clarity (squared) & .052 & .107 & -.747 & .106 \\
FFMQ Nonreactivity & -.070 &. $\mathbf{5 1 4}$ & -.072 & -.093 \\
TMMS Repair (squared) & .188 & .564 & .030 & .047 \\
DERS Goals & -.138 & .533 & -.066 & .042 \\
DERS Impulse Control (squared) & .105 & $\mathbf{. 6 8 3}$ & .015 & .132 \\
DERS Strategies (squared) & .027 &. $\mathbf{9 0 2}$ & .018 & .127 \\
FFMQ Acting with awareness & .026 & .099 & -.121 & .225 \\
\hline
\end{tabular}


Table 4. Exploratory Factor Analysis Excluding the FFMQ Acting with Awareness Subscale (Study 1)

\begin{tabular}{lcrrr}
\hline & \multicolumn{5}{c}{ Factor } \\
\cline { 2 - 5 } Indicator & 1 & 2 & 3 & 4 \\
\hline FFMQ Observe & .357 & .128 & -.020 & -.233 \\
DERS Awareness & .943 & -.146 & -.186 & .185 \\
FFMQ Nonjudging & -.052 & .081 & -.111 & .696 \\
DERS Acceptance (squared) & .110 & .286 & -.092 & .559 \\
FFMQ Describe & .032 & -.086 & -.748 & -.056 \\
TMMS Clarity & -.022 & .123 & -.863 & .067 \\
DERS Clarity (squared) & .057 & .111 & -.745 & .115 \\
FFMQ Nonreactivity & -.076 & .520 & -.071 & -.100 \\
TMMS Repair (squared) & .183 & .572 & .021 & .033 \\
DERS Goals & .026 & .893 & .014 & .153 \\
DERS Impulse Control (squared) & .106 &. $\mathbf{6 8 4}$ & .012 & .144 \\
DERS Strategies (squared) & & & & \\
\hline
\end{tabular}


Table 5. Factor Inter-correlations for Acceptance, Clarity, and Negative Emotion Regulation (Study 1)

\begin{tabular}{llll}
\hline Factor & 1 & 2 & 3 \\
\hline 1. Acceptance & 1.00 & & \\
2. Clarity & 0.62 & 1.00 & \\
3. Negative Emotion Regulation & 0.67 & 0.59 & 1.00 \\
\hline
\end{tabular}


Table 6. Descriptive Statistics and Indicator Inter-correlations (Study 2)

\begin{tabular}{|c|c|c|c|c|c|c|c|c|c|c|c|c|c|c|c|c|c|}
\hline & & Std. & & & & & & & & & & & & & & & \\
\hline Indicator & Mean & Dev. & 1 & 2 & 3 & 4 & 5 & 6 & 7 & 8 & 9 & 10 & 11 & 12 & 13 & 14 & 15 \\
\hline 1. FFMQ Observe & 3.21 & 0.56 & 1 & & & & & & & & & & & & & & \\
\hline 2. FFMQ Nonjudging & 3.36 & 0.71 & -.14 & 1 & & & & & & & & & & & & & \\
\hline $\begin{array}{l}\text { 3. DERS Acceptance } \\
\text { (squared) }\end{array}$ & 3.98 & 0.77 & -.03 & .52 & 1 & & & & & & & & & & & & \\
\hline 4. FFMQ Describe & 3.39 & 0.65 & .21 & .10 & .21 & 1 & & & & & & & & & & & \\
\hline 5. TMMS Clarity & 3.55 & 0.59 & .09 & .33 & .34 & .65 & 1 & & & & & & & & & & \\
\hline 6. DERS Clarity (squared) & 3.93 & 0.64 & .11 & .28 & .32 & .61 & .80 & 1 & & & & & & & & & \\
\hline 7. FFMQ Nonreactivity & 3.06 & 0.52 & .13 & .18 & .14 & .11 & .26 & .24 & 1 & & & & & & & & \\
\hline 8. TMMS Repair (squared) & 3.71 & 0.67 & .13 & .13 & .25 & .14 & .29 & .28 & .23 & 1 & & & & & & & \\
\hline 9. DERS Goals & 2.98 & 0.97 & .01 & .16 & .32 & .09 & .20 & .21 & .20 & .24 & 1 & & & & & & \\
\hline 10. DERS Strategies (squared) & 4.05 & 0.73 & .02 & .37 & .52 & .14 & .39 & .36 & .35 & .52 & .49 & 1 & & & & & \\
\hline 11. DERS Impulse Control (squared) & 4.31 & 0.73 & .00 & .30 & .43 & .13 & .30 & .35 & .34 & .31 & .40 & .64 & 1 & & & & \\
\hline 12. Linking Inventory (reverse-scored) & 0.58 & 0.19 & .05 & .03 & .07 & -.01 & .09 & .07 & .21 & .40 & .23 & .37 & .20 & 1 & & & \\
\hline 13. RRQ Rumination scale & 3.54 & 0.65 & .07 & -.35 & -.36 & -.203 & -.36 & -.30 & -.27 & -.34 & -.38 & -.47 & -.27 & -.28 & 1 & & \\
\hline
\end{tabular}




\begin{tabular}{|c|c|c|c|c|c|c|c|c|c|c|c|c|c|c|c|c|c|}
\hline & & Std. & & & & & & & & & & & & & & & \\
\hline Indicator & Mean & Dev. & 1 & 2 & 3 & 4 & 5 & 6 & 7 & 8 & 9 & 10 & 11 & 12 & 13 & 14 & 15 \\
\hline 14. BSI Depression (square root) & 1.84 & 0.61 & .06 & -.37 & -.35 & -.225 & -.39 & -.37 & -.30 & -.44 & -.22 & -.58 & -.36 & -.28 & .48 & 1 & \\
\hline 15. BSI Anxiety (square root) & 1.76 & 0.56 & .10 & -.35 & -.30 & -.188 & -.30 & -.27 & -.20 & -.25 & -.20 & -.41 & -.32 & -.17 & .35 & .56 & 1 \\
\hline
\end{tabular}

Notes. Correlations $>0.11$ are significant at $\mathrm{p}<0.01$. Means and standard deviations are reported in their original, rather than their transformed, units. 
Table 7. Confirmatory Factor Analysis Standardized Factor Loadings (Study 2)

\begin{tabular}{llll}
\hline & \multicolumn{2}{c}{ Factor } \\
\cline { 2 - 3 } Indicator & Acceptance & Clarity & Negative Emotion \\
& & & Regulation \\
\hline FFMQ Nonjudging & 0.61 & & \\
DERS Acceptance (squared) & 0.83 & 0.69 & \\
FFMQ Describe & & 0.93 \\
TMMS Clarity & & 0.86 \\
DERS Clarity (squared) & & 0.39 \\
FFMQ Nonreactivity & & 0.54 \\
TMMS Repair (squared) & & 0.53 \\
DERS Goals & & 0.92 \\
DERS Impulse Control (squared) & & \\
DERS Strategies (squared) & & \\
\hline
\end{tabular}


Table 8. Factor Inter-correlations for Acceptance, Clarity, and Negative Emotion Regulation (Study 2)

\begin{tabular}{llll}
\hline Factor & 1 & 2 & 3 \\
\hline 1. Acceptance & 1.00 & & \\
2. Clarity & 0.45 & 1.00 & \\
3. Negative Emotion Regulation & 0.66 & 0.44 & 1.00 \\
\hline
\end{tabular}


Table 9. Indirect Effects (Standardized) of Each Factor on Other Factors (Study 2)

\begin{tabular}{|c|c|c|c|c|c|c|c|}
\hline $\begin{array}{l}\text { Recipient of } \\
\text { Effect }\end{array}$ & Attention & Acceptance & Clarity & $\begin{array}{l}\text { Emotion } \\
\text { Regulation }\end{array}$ & $\begin{array}{c}\text { Non- } \\
\text { attachment }\end{array}$ & Rumination & $\begin{array}{c}\text { Psychological } \\
\text { Distress }\end{array}$ \\
\hline Clarity & - & - & - & - & - & - & - \\
\hline $\begin{array}{l}\text { Emotion } \\
\text { Regulation }\end{array}$ & 0.03 & 0.08 & - & - & - & - & - \\
\hline $\begin{array}{l}\text { Non- } \\
\text { attachment }\end{array}$ & 0.03 & 0.51 & 0.12 & - & - & - & - \\
\hline Rumination & -0.04 & -0.17 & -0.05 & -0.17 & - & - & - \\
\hline $\begin{array}{l}\text { Psychological } \\
\text { Distress }\end{array}$ & -0.06 & -0.55 & -0.12 & -0.06 & -0.04 & - & - \\
\hline
\end{tabular}


Table 10. Total Effects (Standardized) of Each Factor on Other Factors (Study 2)

\begin{tabular}{llllcccc}
\hline $\begin{array}{l}\text { Recipient of } \\
\text { Effect }\end{array}$ & Attention & Acceptance & Clarity & $\begin{array}{c}\text { Emotion } \\
\text { Regulation }\end{array}$ & $\begin{array}{c}\text { Non- } \\
\text { attachment }\end{array}$ & $\begin{array}{c}\text { Rumination } \\
\text { Psychological } \\
\text { Distress }\end{array}$ \\
\hline $\begin{array}{l}\text { Clarity } \\
\text { Emotion }\end{array}$ & 0.21 & 0.50 & - & - & - & - & - \\
$\begin{array}{l}\text { Regulation } \\
\text { Non- }\end{array}$ & 0.03 & 0.67 & 0.16 & - & - & - & - \\
$\begin{array}{l}\text { attachment } \\
\text { Rumination }\end{array}$ & -0.04 & $0.08(n s)$ & 0.12 & 0.77 & - & - & - \\
$\begin{array}{l}\text { Psychological } \\
\text { Distress }\end{array}$ & 0.07 & -0.54 & -0.19 & -0.28 & -0.22 & - & - \\
\hline
\end{tabular}

Note. "ns" denotes total effects that are not statistically significantly different from zero. 
Table 11. Total Effects (Standardized) of Each Factor on Other Factors when Both Psychological Distress and Positive Psychological Adjustment are Included in the Model (Study 2)

\begin{tabular}{|c|c|c|c|c|c|c|c|c|}
\hline $\begin{array}{l}\text { Recipient of } \\
\text { Effect }\end{array}$ & Attention & Acceptance & Clarity & $\begin{array}{c}\text { Emotion } \\
\text { Regulation }\end{array}$ & $\begin{array}{c}\text { Non- } \\
\text { attachment }\end{array}$ & Rumination & $\begin{array}{l}\text { Psychological } \\
\text { Distress }\end{array}$ & $\begin{array}{c}\text { Positive } \\
\text { Psychological } \\
\text { Adjustment }\end{array}$ \\
\hline Clarity & 0.21 & 0.51 & - & - & - & - & - & - \\
\hline $\begin{array}{l}\text { Emotion } \\
\text { Regulation }\end{array}$ & 0.03 & 0.68 & 0.15 & - & - & - & - & - \\
\hline $\begin{array}{l}\text { Non- } \\
\text { attachment }\end{array}$ & 0.03 & 0.07 (ns) & 0.12 & 0.81 & - & - & - & - \\
\hline Rumination & -0.04 & -0.56 & -0.18 & -0.23 & -0.28 & - & - & - \\
\hline $\begin{array}{l}\text { Psychological } \\
\text { Distress }\end{array}$ & $\begin{array}{l}0.06 \\
(n s)\end{array}$ & -0.55 & -0.31 & -0.60 & -0.12 & 0.19 & - & - \\
\hline $\begin{array}{l}\text { Positive } \\
\text { Psychological } \\
\text { Adjustment }\end{array}$ & 0.18 & 0.40 & 0.29 & 0.70 & 0.25 & -0.15 & - & - \\
\hline
\end{tabular}

Note. "ns" denotes total effects that are not statistically significantly different from zero. 


\section{FIGURES}

Figure 1. Confirmatory Factor Analysis for Four Factors Identified in Exploratory Factor Analysis (Study 1)

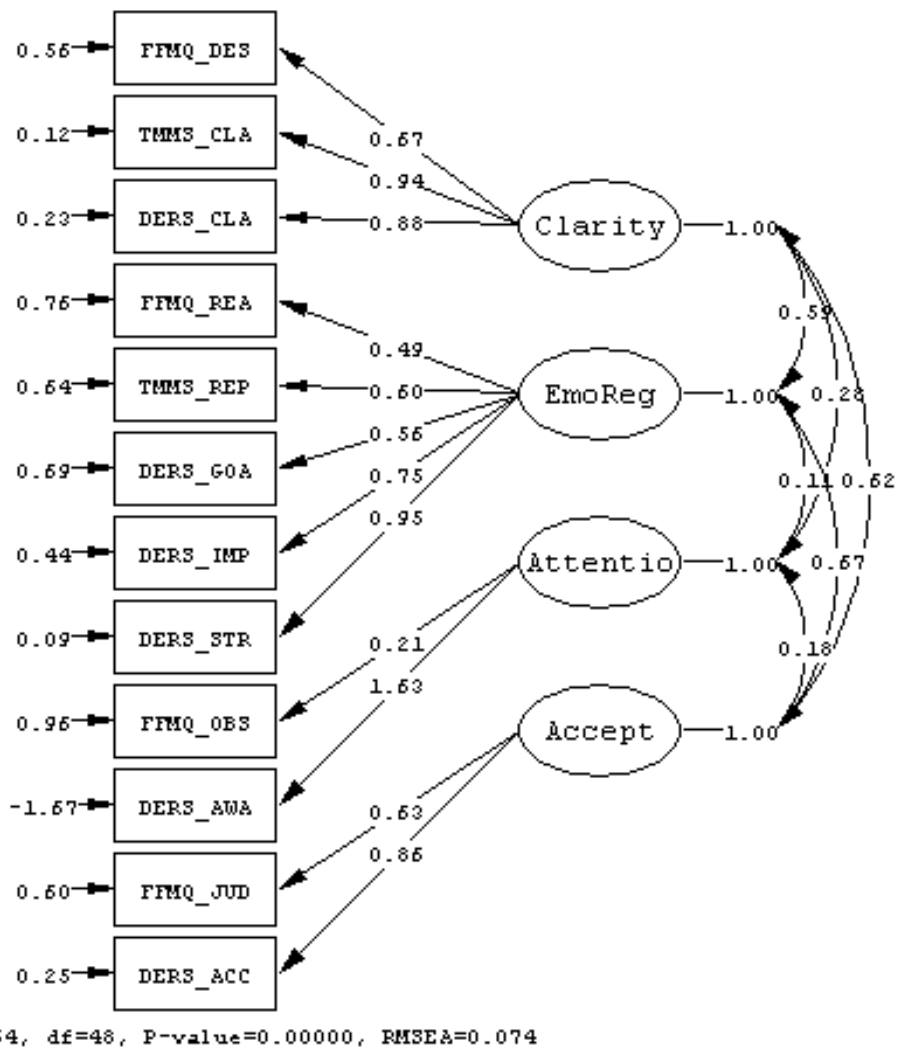


Figure 2. Confirmatory Factor Analysis for Acceptance, Clarity, and Negative Emotion Regulation (Study 1)
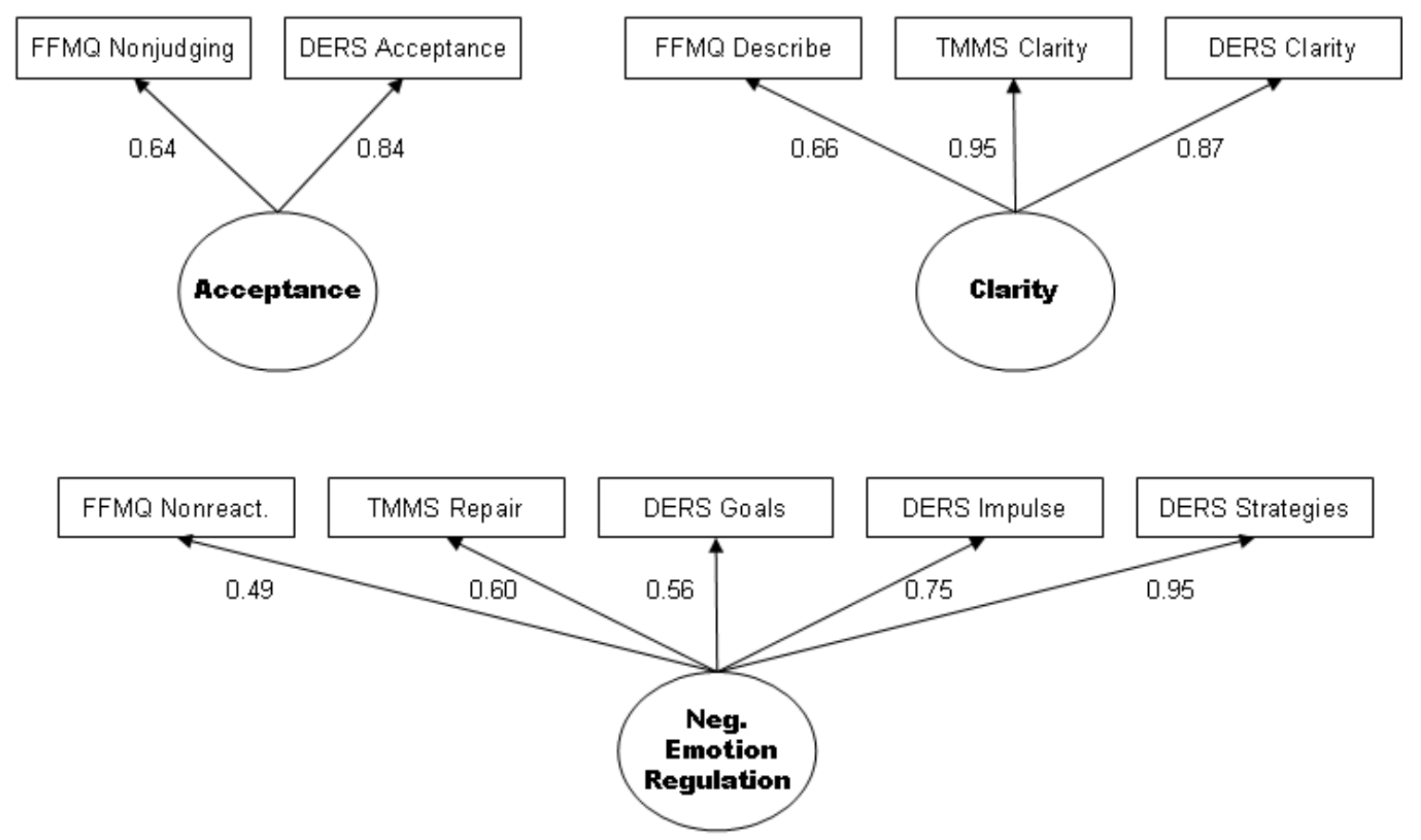

RMSEA $=0.055$

$X_{2}=70.52 ; d f=32$

$p=0.0001$ 
Figure 3. Relationships among the Factors (Study 1)

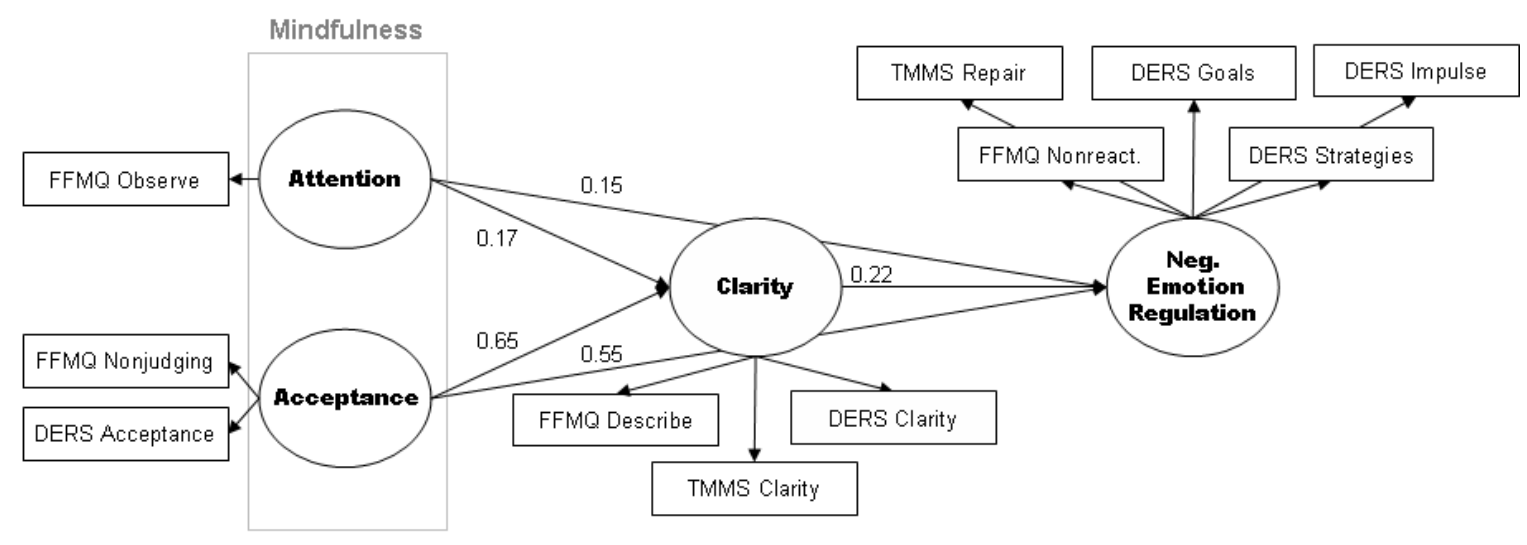

RMSEA $=0.059$

$\chi^{2}=93.84 ; d f=39$

$p<0.0001$ 
Figure 4. Full Model with All Possible Pathways (Study 2)

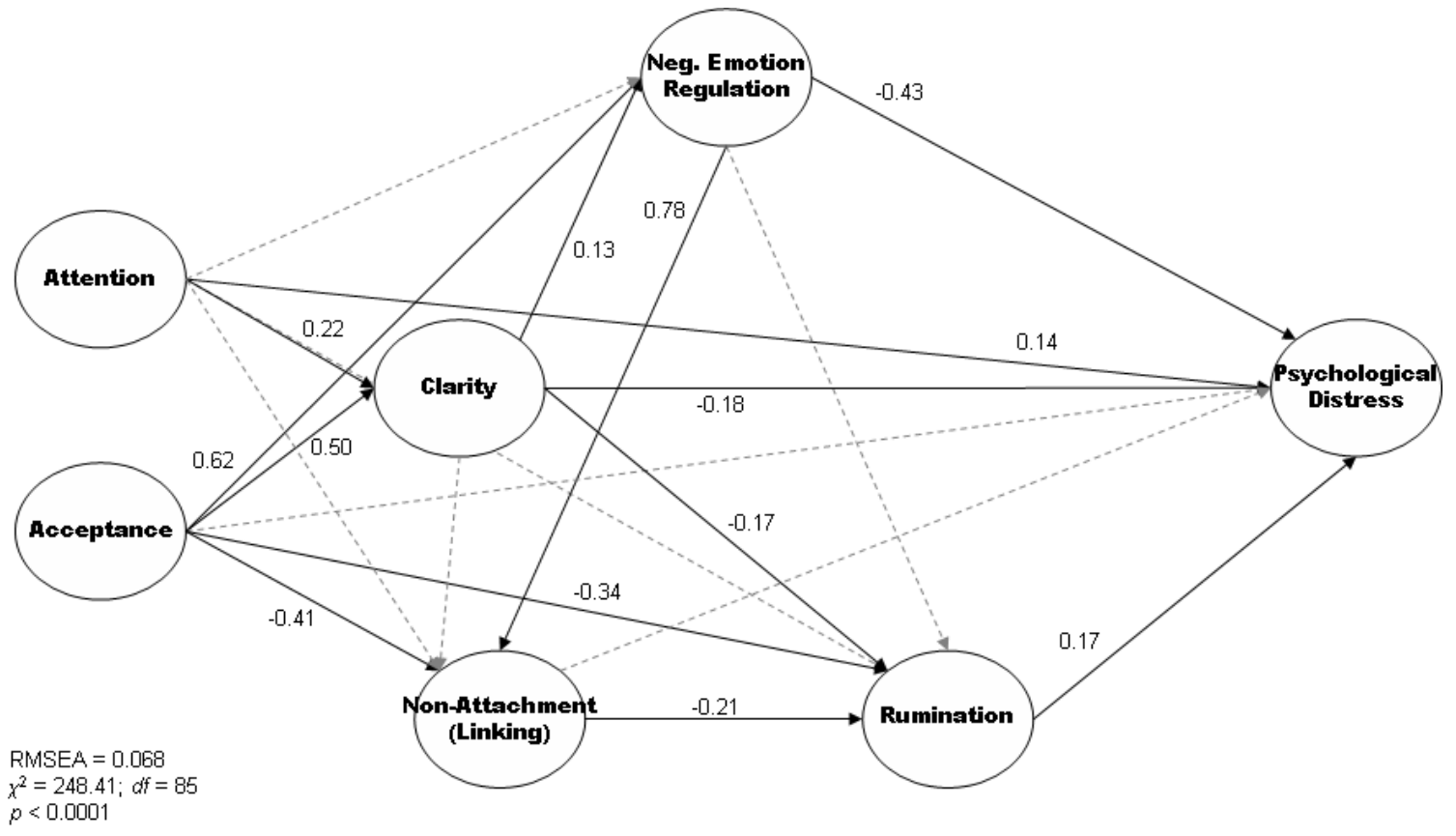


Figure 5. Full Model with All Hypothesized or Significant pathways (Study 2)

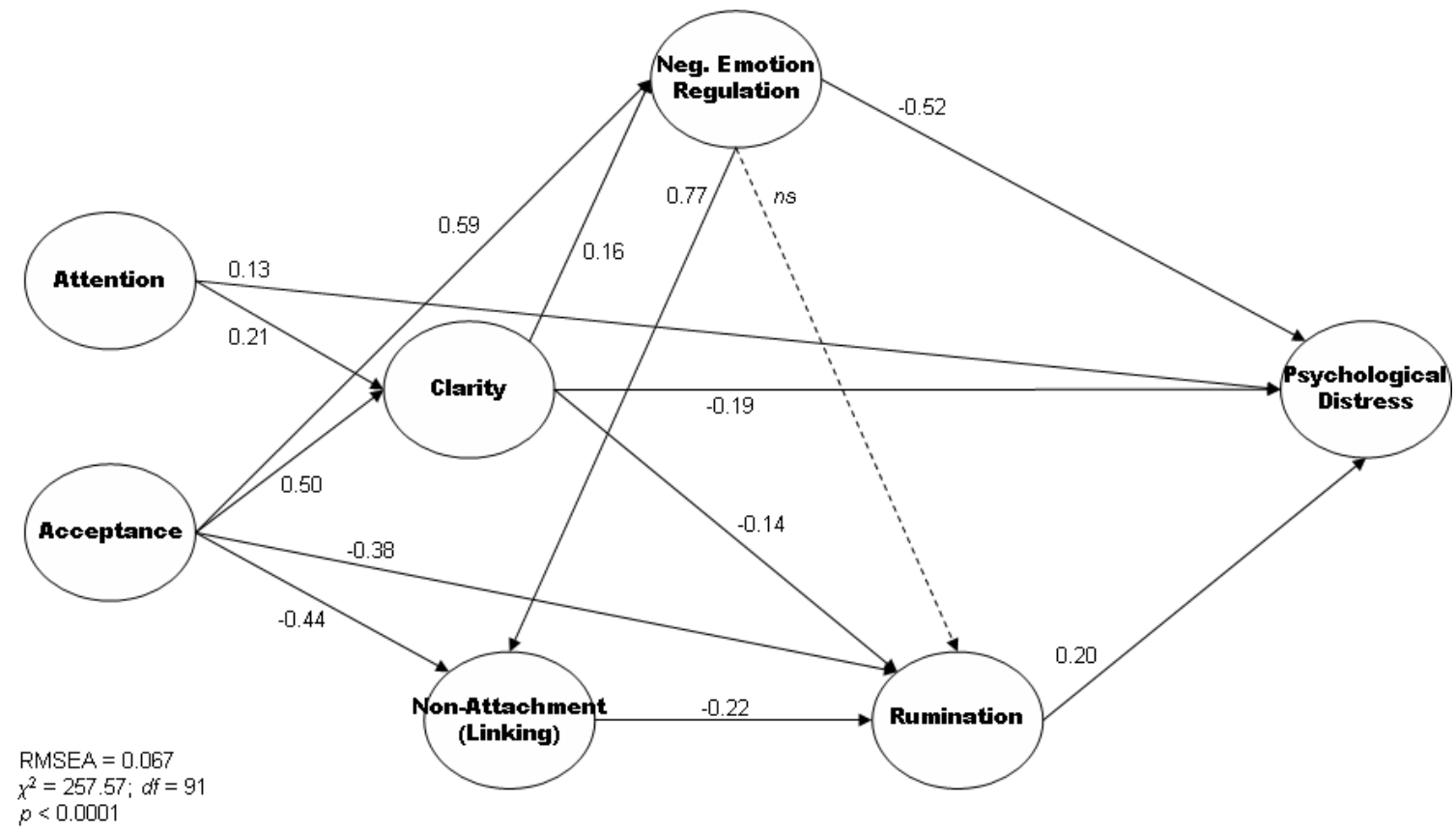


Figure 6. Full Model with Flourishing Mental Health as the Final Variable and all Possible Pathways (Study 2)

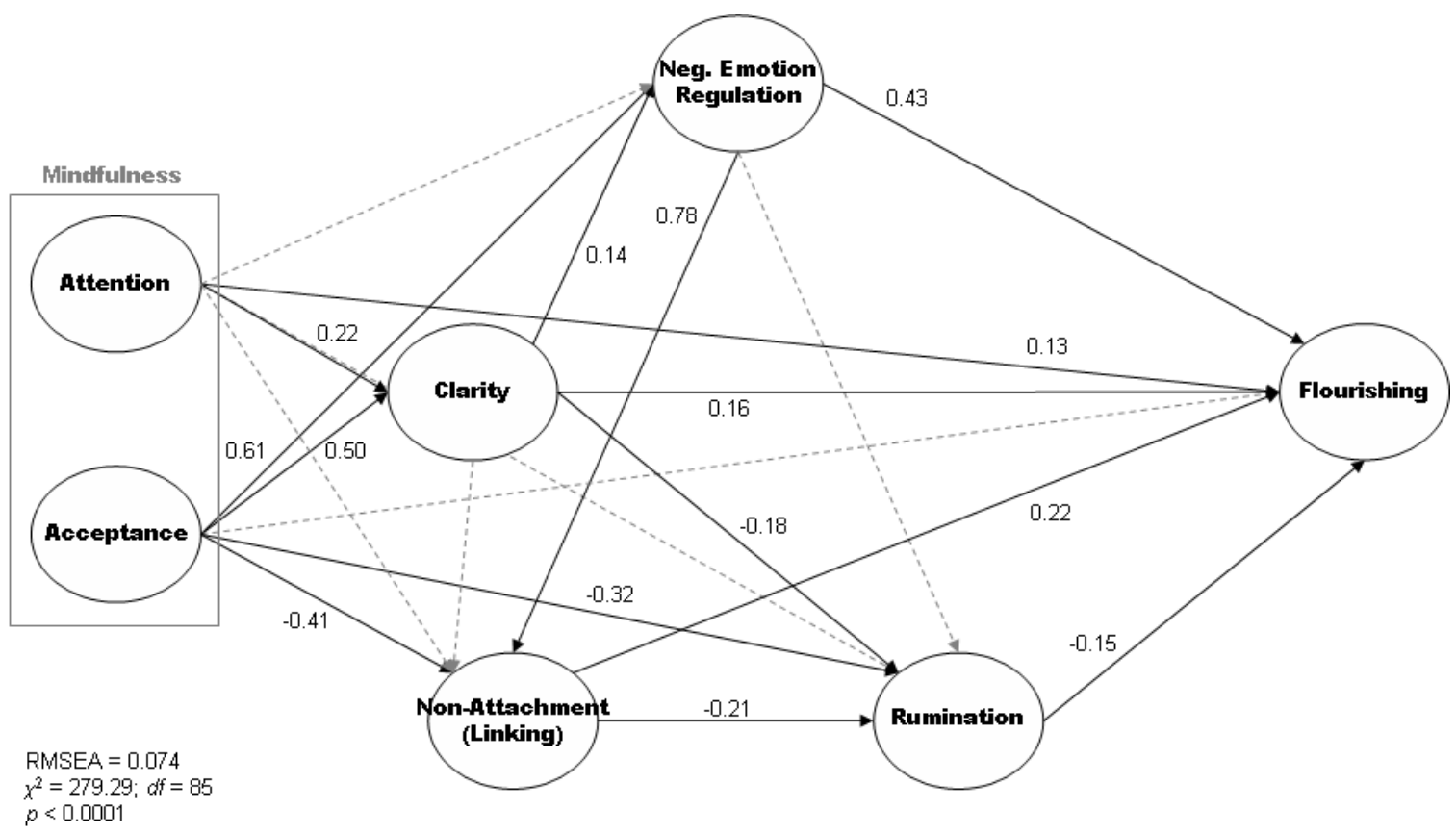


Figure 7. Full Model with Flourishing Mental Health as the Final Variable and all

Significant Pathways (Study 2)

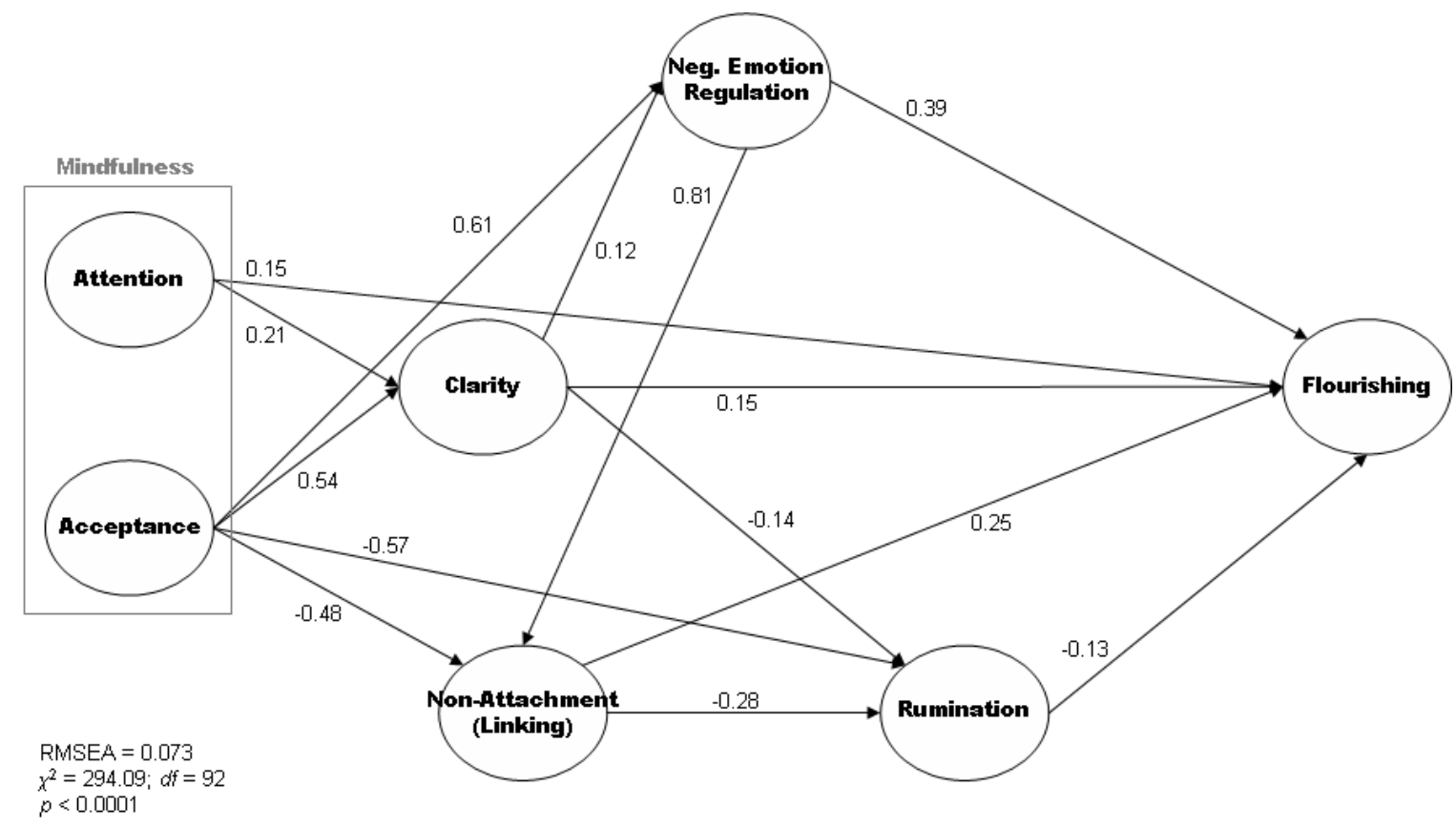


Figure 8. Full Model with Both Psychological Distress and Flourishing Mental Health as

Outcome Variables (Study 2)

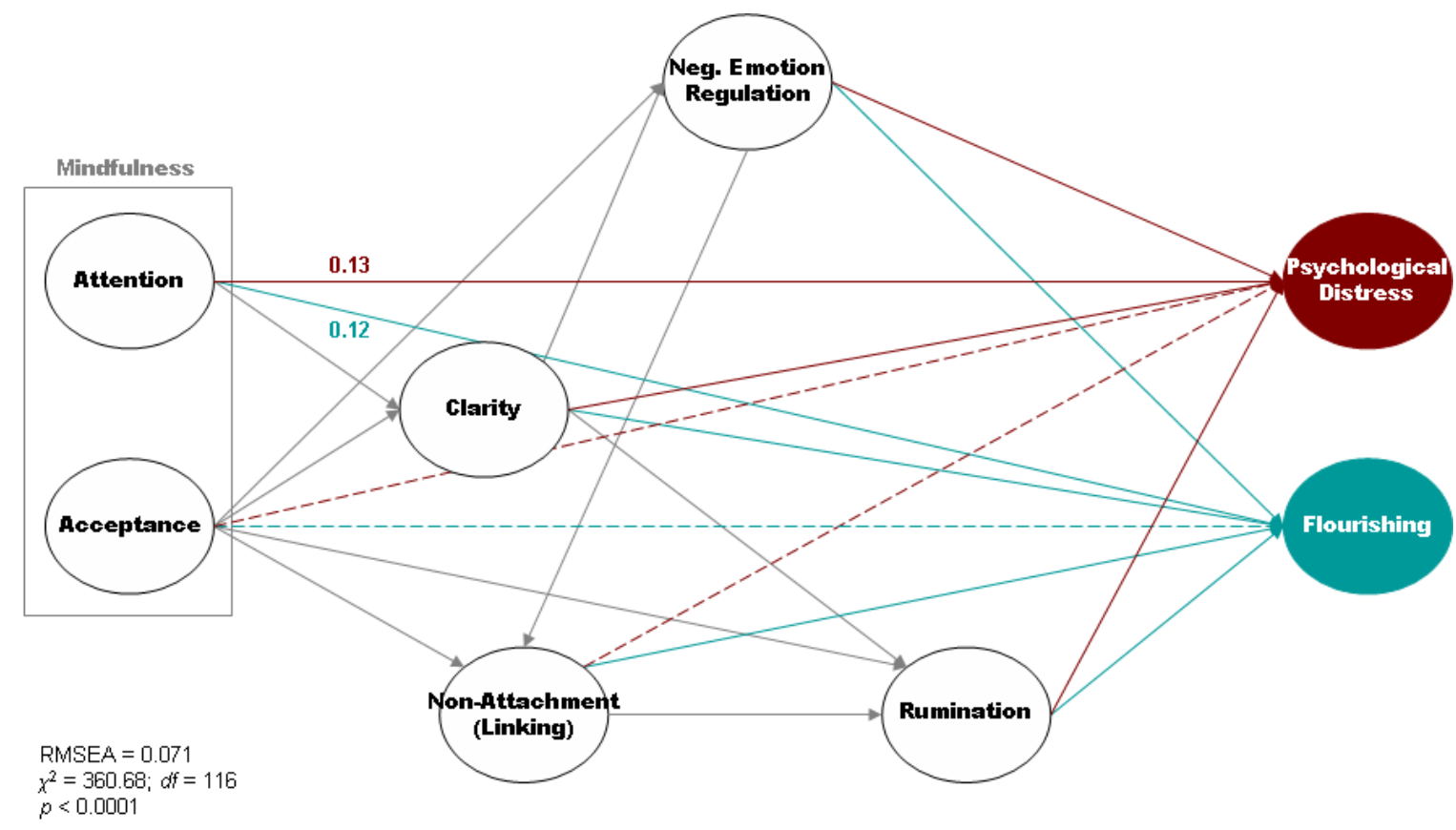


Figure 9. Exploratory Model 1: Incorporating the FFMQ Acting with Awareness Subscale into the Model (Study 1)

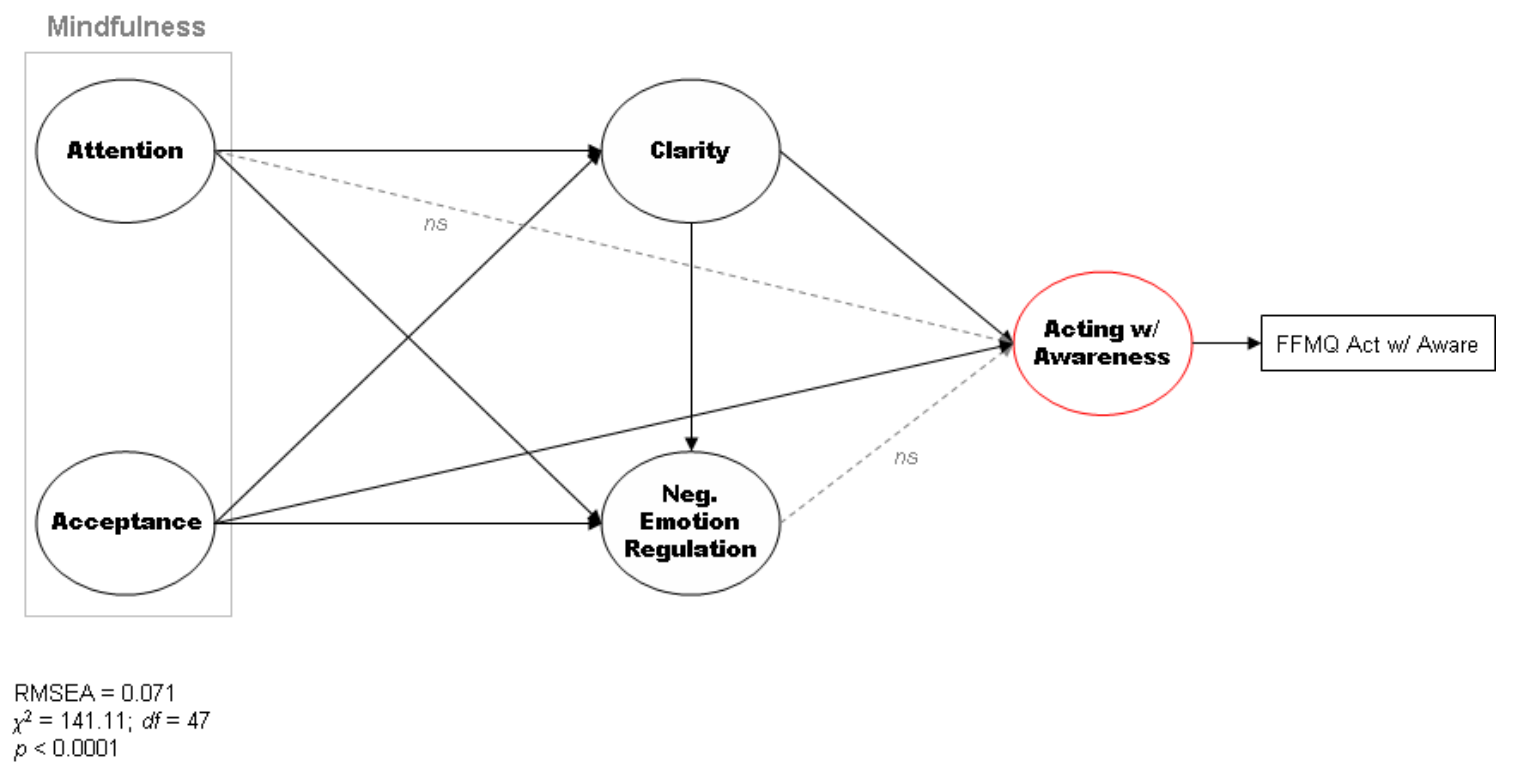


Figure 10. Exploratory Model 2: Incorporating the FFMQ Acting with Awareness Subscale into the Model (Study 1)

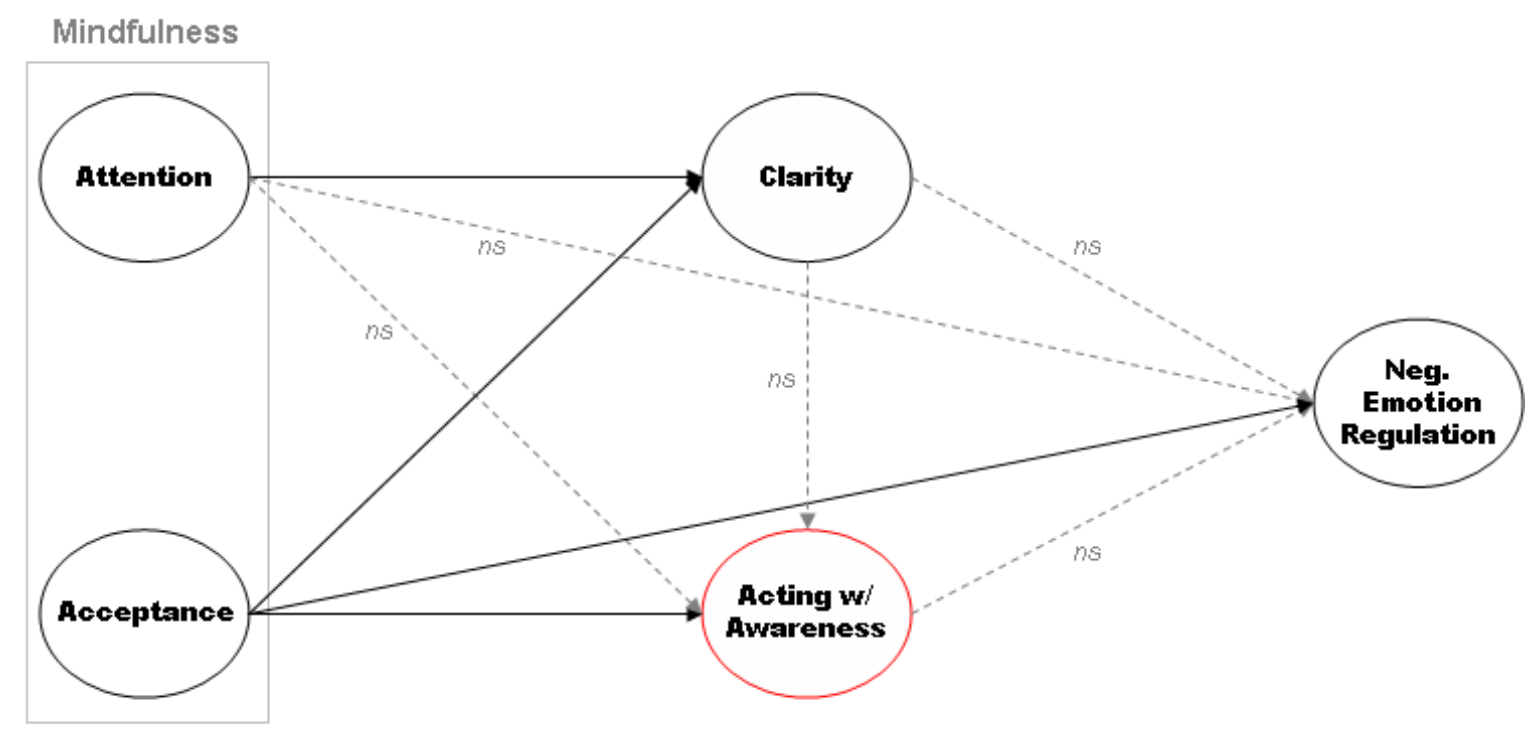

RMSEA $=0.083$

$\chi^{2}=169.49 ; d f=46$

$p<0.0001$ 
Figure 11. Exploratory Model 3: Incorporating the FFMQ Acting with Awareness Subscale into the Model (Study 1)

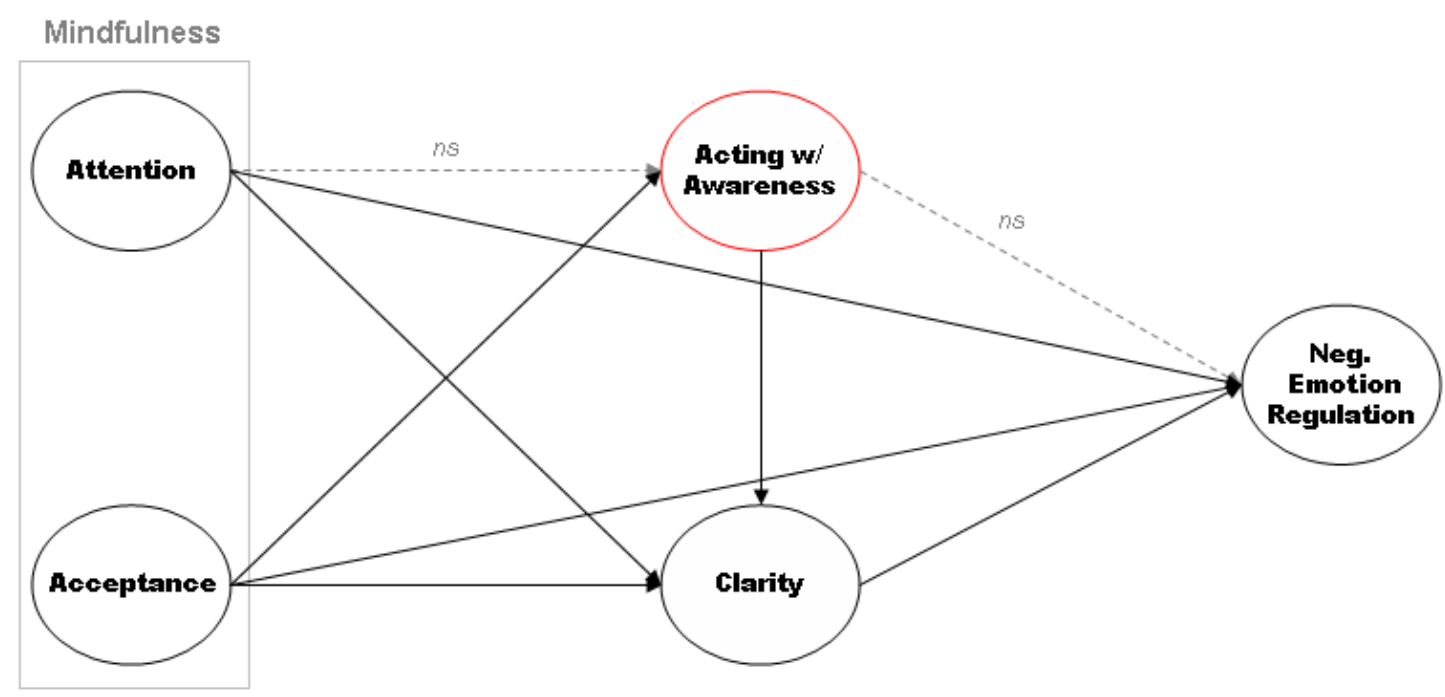

RMSEA $=0.070$

$x^{2}=135.07 ; d f=46$

$p<0.0001$ 
Figure 12. Exploratory Model 4: Incorporating the FFMQ Acting with Awareness Subscale into the Model (Study 1)

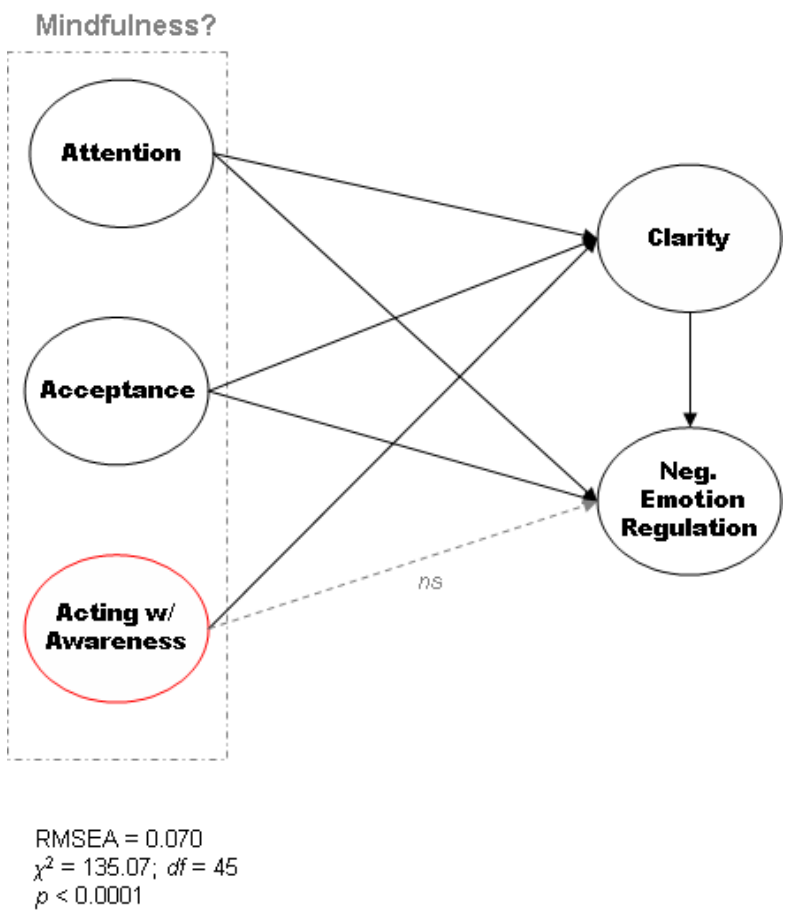




\section{REFERENCES}

Arch, J.J. \& Craske, M.G. (2006). Mechanisms of mindfulness: Emotion regulation following a focused breathing induction. Behaviour Research and Therapy, 44, 18491858.

Baer, R.A. (2003). Mindfulness training as a clinical intervention: A conceptual and empirical review. Clinical Psychology: Science and Practice, 10, 125-143.

Baer, R.A., Smith, G.T., \& Allen, K.B. (2004). Assessment of mindfulness by self-report: The Kentucky inventory of mindfulness skills. Assessment, 11(3), 191-206.

Baer, R.A., Smith, G.T., Hopkins, J., Krietemeyer, J., \& Toney, L. (2006). Using self-report assessment methods to explore facets of mindfulness. Assessment, 13(1), 27-45.

Begley, S. (2007, January 19). How the brain rewires itself. Time, 169:5, 72-79.

Bishop, S.R., Lau, M., Shapiro, S., Carlson, L., Anderson, N.D., Carmody, J., Segal, Z.V., Abbey, S., Speca, M., Velting, D., \& Devins, G. (2004). Mindfulness: A proposed operational definition. Clinical Psychology: Science and Practice, 11(3), 230-241.

Boden, J.M., Hyland, M.E., \& Dale, K.L. (2005). Defensiveness and symptom reporting. Current Psychology: Developmental • Learning • Personality• Social, 24, 153-170.

Breslin, F.C., Zack, M., \& McMain, S. (2002). An information-processing analysis of mindfulness: Implications for relapse prevention in the treatment of substance abuse. Clinical Psychology: Science and Practice, 9, 275-299.

Brown, K.W. \& Ryan, R.M. (2003). The benefits of being present: Mindfulness and its role in psychological well-being. Journal of Personality and Social Psychology, 84 (4), 822848.

Brown, K. W. \& Ryan, R.M. (2004). Perils and promise in defining and measuring mindfulness: Observations from experience. Clinical Psychology: Science and Practice, 11 (3), 242-248.

Brown, K. W., Ryan, R.M., \& Creswell, J.D. (2007). Mindfulness: Theoretical foundation and evidence for its salutary effects. Psychological Inquiry, 18 (4), 211-237.

Buchheld, N., Grossman, P., \& Wallach, H. (2001). Measuring mindfulness in insight meditation (Vipassana) and meditation-based psychotherapy: The development of the Freiburg Mindfulness Inventory (FMI). Journal for Meditation and Meditation Research, $1,11-34$.

Carson, J.W., Carson, K.M., Gil, K.M., Baucom, D.H. (2004). Mindfulness-based relationship enhancement. Behavior Therapy, 35, 471-494. 
Chadwick, P., Hember, M., Mead, S., Lilley, B., \& Dagnan, D. (2005). Responding mindfully to unpleasant thoughts and images: Reliability and validity of the Mindfulness Questionnaire. Unpublished manuscript.

Coffey, K.A. \& Hartman, M. (2008). Mechanisms of action in the inverse relationship between mindfulness and psychological distress. Complementary Health Practice Review, 13, 79-91.

Crowne, D.P. \& Marlowe, D. (1960). A new scale of social desirability independent of psychopathology. Journal of Consulting Psychology, 24, 349-354.

Cullen, L.T. (2006, January 16). How to get smarter, one breath at a time. Time, 167:3, 93.

Dalai Lama \& Cutler, H.C. (1998). The Art of Happiness: A Handbook for Living. New York: Riverhead Books.

Davidson, R.J., Kabat-Zinn, J., Schumacher, J., Rosenkranz, M., Muller, D., Santorelli, S., Urbanowski, F., Harrington, A., Bonus, K., \& Sheridan, J.F. (2003). Alterations in brain and immune function produced by mindfulness meditation. Psychosomatic Medicine, 65 (4), 564-570.

Deikman, A. (1966). Deautomatization and the Mystic Experience. Psychiatry, 29, 324-388.

Derogatis, L. (1977). The SCL-90-R: Administration, Scoring, and Procedures Manual 1, Clinical Psychometric Research, Baltimore.

Derogatis, L. (1983). The brief symptom inventory: An introductory report. Psychological Medicine, 13 (3), 595-605.

Diener, E., Emmons, R. A., Larsen, R. J., \& Griffin, S. (1985). The Satisfaction with Life Scale. Journal of Personality Assessment, 49, 71-75.

Dimidjian, S. \& Linehan, M. (2003). Defining an agenda for future research on the clinical applications of mindfulness practice. Clinical Psychology: Science and Practice, 10, 166171.

Ekblad, A. G., Smoski, M., Keng, S. L., Robins, C. J., Brantley, J. B., \& Lynch, T. R. (In preparation). Emotional and physiological effects of a mindful breathing exercise in mindfulness-naïve individuals.

Ekman, P., Davidson, R.J., Ricard, M., \& Wallace, B.A. (2005) Buddhist and psychological perspectives on emotions and well-being. Current Directions in Psychological Science, 14 (2), 59-63.

Feldman, G.C., Hayes, A.M., Kumar, S.M., \& Greeson, J.M. (2004). Development, factor structure, and initial validation of the Cognitive and Affective Mindfulness Scale. Unpublished manuscript. 
Garfinkel, Perry. (2006, April). Buddha rising. National Geographic, 208, 88-109.

Gratz, K.L. \& Roemer, L. (2004). Multidimensional assessment of emotion regulation and dysregulation: Development, factor structure, and initial validation of the difficulties in emotion regulation scale. Journal of Psychopathology and Behavioral Assessment, 26(1), $41-54$.

Gross, J.J. (1998). The emerging field of emotion regulation: An integrative review. Review of General Psychology, 2, 271-299.

Goldstein, J. (1976). The experience of insight. Boston: Shambhala.

Goleman, D. (2003). Destructive Emotions: A Scientific Dialogue with the Dalai Lama. New York, New York: Bantam Dell.

Hanh, T. N. (1998). The Heart of the Buddha's Teaching. Berkeley, CA: Parallax Press.

Hayes, S.C., Strosahl, K.D., \& Wilson, K.G. (1999). Acceptance and commitment therapy: An experiential approach to behavior change. New York: Guildford Press.

Jain, S., Shapiro, S.L., Swanick, S., Roesch, S.C., Mills, P.J., Bell, I., and Schwartz, G.E.R. (2007). A randomized controlled trial of mindfulness meditation versus relaxation training: Effects on distress, positive states of mind, rumination, and distraction. Annals of Behavioral Medicine, 33, 11-21.

Jenkins, L. (2007, February 12). Deep breath as pitchers rethink routines. New York Times, p. D1.

Jöreskog, K. \& Sörbom, D. (2001). LISREL 8.51: User's reference guide. Chicago: Scientific Software International.

Kabat-Zinn, J. (1990). Full Catastrophe Living. New York, New York: Dell Publishing.

Kabat-Zinn, J. (1994). Wherever you go, there you are: Mindfulness meditation in everyday life. New York: Hyperion.

Kabat-Zinn, J. (2003). Mindfulness-based interventions in context: Past, present, and future. Clinical Psychology: Science and Practice, 10, 144-145.

Kabat-Zinn, J., Massion, A.O., Kristeller, J., Peterson, L.G. et al. (1992). Effectiveness of a meditation-based stress reduction program in the treatment of anxiety disorders. American Journal of Psychiatry, 149 (7), 936-943.

Kalb. C. (2004, October 3). Buddha lessons. Newsweek, 144:13, 48-51.

Keyes, C. (2005). The subjective well-being of America's Youth: Toward a comprehensive assessment. Adolescent and Family Health, 4, 3-11. 
Kristeller, J.L., \& Hallett, C.B. (1999). An exploratory study of a meditation-based intervention for binge eating disorder. Journal of Health Psychology, 4, 357-363.

Kyabgon, T. (2001). The Essence of Buddhism. Boston: Shambhala.

Linehan, M.M. (1993). Cognitive-behavioral treatment of borderline personality disorder. New York: The Guilford Press.

Linehan, M.M., Armstrong, H.E., Suarez, A., Allmon, D., \& Heard, H.L. (1991). A cognitive-behavioral treatment of chronically parasuicidal borderline patients. Archives of General Psychiatry, 48, 1060-1064.

Martin, L.L., \& Tesser, A. (1989). Toward a motivational and structural theory of ruminative thought. In J.S. Uleman \& J.A. Bargh (Eds.), Unintended thought: The limits of awareness, intention, and control (pp. 552-572). New York: Guilford.

McIntosh, W.D. (1997). East meets West: Parallels between Zen Buddhism and social psychology. International Journal for Psychology of Religion, 7, 37-52.

McIntosh, W.D., Harlow, T.F., and Martin, L.L. (1995). Linkers and nonlinkers: Goal beliefs as a moderator of the effects of everyday hassles on rumination, depression, and physical complaints. Journal of Applied Social Psychology, 25, 1231-1244.

McIntosh, W.D., and Martin, L.L. (1992). The cybernetics of happiness: The relation between goal attainment, rumination, and affect. In M.S. Clark (Ed.), Review of personality and social psychology (Vol. 14, pp. 222-246). Newbury Park, CA: Sage.

McLean, P.D. \& Woody, S.R. (2001). Anxiety disorders in adults: An evidence-based approach to psychological treatment. New York: Oxford University Press.

Nolen-Hoeksema, S. (1991). Responses to depression and their effects on the duration of depressive episodes. Journal of Abnormal Psychology, 100, 569-582.

Nolen-Hoeksema, S. (2000). The role of rumination in depressive disorders and mixed anxiety/depression symptoms. Journal of Abnormal Psychology, 109, 504-511.

Ornish, Dean. (2006, August 14). How to stress less. Newsweek, 148:7, 60.

Pradhan, E.K., Baumgarten, M., Langenberg, P., Handwerger, B., Gilpin, A.K., Magyari, T., Hochberg, M.C., \& Berman, B.M. (2007). Effects of mindfulness-based stress reduction in rheumatoid arthritis patients. Arthritis \& Rheumatism, 57, 1134-1142.

Roemer, L. \& Orsillo, S.M. (2003). Mindfulness: A promising intervention strategy in need of further study. Clinical Psychology: Science and Practice, 10, 172-178.

Salovey, P., Mayer, J.D., Goldman, S., Turvey, C., and Palfai, T. (1995). Emotional attention, clarity, and repair: exploring emotional intelligence using the trait meta-mood 
scale. In: Pennebaker, J.D. (Ed.), Emotion, Disclosure, and Health, pp. 125-154. Washington, D.C.: American Psychological Association.

Segal, Z.V., Williams, J.M.G., \& Teasdale, J.D. (2002). Mindfulness-based cognitive therapy for depression: A new approach to preventing relapse. New York: Guilford.

Shapiro, S.L., Carlson, L.E., Astin, J.A., \& Freedman, B. (2006). Mechanisms of mindfulness. Journal of Clinical Psychology, 62, 373-386.

Shapiro, S.L., Schwartz, G.E., \& Bonner, G. (1998). Effects of mindfulness-based stress reduction on medical and premedical students. Journal of Behavioral Medicine, 21, 581599.

Siegle, G.J., Moore, P.M., \& Thase, M.E. (2004). Rumination: One construct, many features in health individuals, depressed individuals, and individuals with lupus. Cognitive Therapy and Research, 28, 645-668.

Smallwood, J. \& Schooler, J.W. (2006). The restless mind. Psychological Bulletin, 132, 946958.

Speca, M., Carlson, L.E., Goodey, E., \& Angen, M. (2000). A randomized, wait-list controlled clinical trial: The effect of a mindfulness meditation-based stress reduction program on mood and symptoms of stress in cancer patients. Psychosomatic Medicine, $62,613-622$.

Teasdale, J.D. (1999). Metacognition, mindfulness, and the modification of mood disorders. Clinical Psychology and Psychotherapy, 6, 146-155.

Teasdale, J.D., Segal, Z.V., \& Williams, M.G. (1995). How does cognitive therapy prevent depressive relapse and why should attentional control (mindfulness) training help? Behavior Research and Therapy, 33, 25-39.

Teasdale, J.D., Segal, Z.V., Williams, J.M.G., Ridgeway, V.A., Soulsby, J.M. and Lau, M.A. (2000). Prevention of relapse/recurrence in major depression by mindfulness-based cognitive therapy. Journal of Consulting and Clinical Psychology, 68, 615-623.

Trapnell, P.D. \& Campbell, J.D. (1999). Private self-consciousness and the five-factor model of personality: Distinguishing rumination from reflection. Journal of Personality and Social Psychology, 76 (2), 284-304.

Walach, H., Buchheld, N., Buttenmuller, V., Kleinknecht, N., \& Schmidt, S. (2006). Measuring mindfulness - the Freiburg Mindfulness Inventory. Personality and Individual Differences, 40, 1543 - 1555.

Zettle, R.D. (2003). Acceptance and commitment therapy vs. systematic desensitization in treatment of mathematics anxiety. Psychological Record, 53 (2), 197-215. 
Zylowska, L., Ackerman, D.L., Yang, M.H., Futrell, J.L., Horton, N.L., Hale, T.S., Pataki, C., \& Samlley, S.L. (2006). Behavioral and cognitive changes in Attention Deficit Hyperactivity Disorder using a mindfulness meditation approach. Manuscript submitted for publication. 